\title{
Neutrino absorption by hot nuclei in supernova environments
}

\author{
Alan A. Dzhioev, ${ }^{1, *}$ A. I. Vdovin, ${ }^{1, \dagger}$ and J. Wambach ${ }^{2,3, \ddagger}$ \\ ${ }^{1}$ Bogoliubov Laboratory of Theoretical Physics, JINR, 141980, Dubna, Russia \\ ${ }^{2}$ Institut für Kernphysik, Technische Universität Darmstadt, 64289 Darmstadt, Germany \\ ${ }^{3}$ GSI Helmholtzzentrum für Schwerionenforschung, Planckstr. 1, 64291 Darmstadt, Germany
}

(Dated: July 6, 2018)

\begin{abstract}
Using the thermal quasiparticle random phase approximation, we study the process of neutrino and antineutrino capture on hot nuclei in supernova environments. For the sample nuclei ${ }^{56} \mathrm{Fe}$ and ${ }^{82} \mathrm{Ge}$ we perform a detailed analysis of thermal effects on the strength distribution of allowed Gamow-Teller transitions which dominate low-energy charged-current neutrino reactions. The finite temperature cross sections are calculated taking into account the contributions of both allowed and forbidden transitions. The enhancement of the low-energy cross sections is explained by considering thermal effects on the $\mathrm{GT}_{ \pm}$strength. For ${ }^{56} \mathrm{Fe}$ we compare the calculated finite-temperature cross sections with those obtained from large-scale shell-model calculations.

PACS numbers: 26.50.+x, 21.60.Jz, 24.10.Pa, 25.30.Pt
\end{abstract}

\section{INTRODUCTION}

It is well known that core-collapse supernova simulations require a detailed description of neutrino transport including all potentially important neutrino reactions [1]. Neutrinos are the mediators of the energy transfer from the core to the outer stellar layers and their luminosities and spectra are a crucial ingredient for the supernova explosion mechanism. Despite significant progress in our understanding of the core-collapse mechanism, many supernova simulations fail to produce explosion (see e.g. $[2,3]$ and references therein). One of the possible reasons for this failure could be the incomplete and inaccurate treatment of the neutrino-nucleus processes occurring in supernova environments.

It was pointed by Haxton [4] that neutral- and chargedcurrent neutrino reactions on nuclei involving the excitation of the giant resonances might help reenergize the explosion and they should, therefore, be taken into account in supernova simulations. Such simulations were performed by Bruenn and Haxton [5] and it was shown that inelastic neutrino-nucleus scattering plays an important role in equilibrating neutrinos with matter. However, the simulations did not confirm Haxton's suggestion that neutrino-nucleus reactions might help to revive the stalled shock wave.

In [5], the nuclear composition of the core was approximated by a single representative nucleus, ${ }^{56} \mathrm{Fe}$, and the relevant cross sections and rates were evaluated considering only allowed (Gamow-Teller) and first-forbidden upward transitions from the nuclear ground state. However, in the hot supernova environment with temperatures $T \gtrsim 1 \mathrm{MeV}$ nuclei exist as a thermal ensemble, i.e., nuclear excited states are thermally populated according

\footnotetext{
*dzhioev@theor.jinr.ru

† vdovin@theor.jinr.ru

‡ jochen.wambach@physik.tu-darmstadt.de
}

to the Boltzmann distribution. As was first realized in Ref. [6], downward transitions from excited states remove the reaction threshold and can significantly enhance the reaction cross section at low neutrino energies.

From a microscopic point of view, there are two ways how to treat neutrino reactions with hot nuclei. The first one involves a state-by-state summation over Boltzmannweighted, individually determined contributions from nuclear ground and excited states. To apply this method, one needs to know the strength distribution of electroweak transition operators for thermally populated nuclear states. The second method is based on a statistical formulation of the nuclear many-body problem. In this approach rather than computing individual strength distributions one determines an "average" temperaturedependent strength function.

For iron group nuclei $(A=45-65)$ the first approach was developed in [7-9] on the basis of large-scale shellmodel (LSSM) diagonalization calculations. Modern high-performance compute capabilities combined with state-of-the-art diagonalization approaches are able to provide detailed strength distributions for both chargeneutral and charge-changing Gamow-Teller transitions that dominate neutrino reactions with $p f$-shell iron group nuclei at low neutrino energies $\left(E_{\nu} \lesssim 20 \mathrm{MeV}\right)$. However, at temperatures $T \gtrsim 1 \mathrm{MeV}$ an explicit state-by-state summation over all thermally populated states presently remains computationally prohibitive. To overcome this problem the "Brink hypothesis" is applied, i.e., it is assumed that GT distributions on nuclear excited states are the same as for the ground state. To account for thermal effects, the giant resonances built on the ground and low-lying daughter states in the inverse reaction are considered. Such excited states are called back-resonances and their importance arises from the large nuclear matrix elements and increased phase space. Thus, within LSSM calculations the temperature dependence of the cross sections is comprised solely in the back-resonance terms.

Within the shell model approach it is assumed that 
the GT distributions on thermally excited states are the same as for the ground state. This is not likely the case as the vanishing of pairing correlations and smearing of the Fermi surface with increasing temperature should affect the distribution centroid and move it slightly down in energy. This conjecture is confirmed by shell model Monte Carlo studies performed at finite temperature [10]. In addition, with present compute capabilities the shell-model diagonalization cannot be applied for nuclei beyond the $p f$-shell because of the huge configurational spacse involved. These shortcomings can be avoided in a thermal quasiparticle random-phase approximation (TQRPA). In [11], the TQRPA was proposed as a method to study the response of hot nuclei to weak external perturbations in the framework of a statistical approach. Being based on the thermo-field dynamics (TFD) formalism [12-14], the TQRPA enables the computation of temperature-dependent strength function avoiding the assumption of Brink's hypothesis. In [15, 16], the TQRPA was applied to study thermal effects on neutral-current inelastic neutrino scattering on ${ }^{54,56} \mathrm{Fe}$ and neutron-rich germanium isotope ${ }^{82} \mathrm{Ge}$. It was shown that the TQRPA reveals the same thermal effects on the cross sections as the LSSM approach. Namely, the reaction threshold for inelastic neutrino-nucleus scattering is removed at finite temperature and the cross section for low-energy neutrinos is significantly enhanced. It was found, however, that within the TQRPA the enhancement is caused by both de-excitation of nuclear excited states and thermally unblocked low-energy GT transitions. The latter do not appear within the LSSM due to application of Brink's hypothesis. Moreover, it was shown in [15] that, unlike in the LSSM approach, the principle of detailed balance is not violated within the TQRPA and it results in a larger strength for downward GT transitions from excited states. As a consequence, at low neutrino energies the finite temperature cross sections calculated within the TQRPA turn out to be several times larger than those obtained within the shell-model calculations.

In the present paper, we apply the TQRPA method to study thermal effects on charged-current neutrinonucleus reactions $\left(\nu_{e}, e^{-}\right)$and $\left(\bar{\nu}_{e}, e^{+}\right)$occurring in the supernova environment. Here we would like to mention that the TQRPA was already applied for chargechanging reactions with hot nuclei when studying thermal effects on electron capture in supernovae [17, 18]. The paper is organized as follows: in Sec. II we review the basics of the TFD formalism and outline how to treat charge-changing transitions in a hot nucleus within the TQRPA. For more details of the approach, the reader is referred to Refs. $[17,18]$. In addition, in Sec. II the expressions necessary to calculate cross-sections for $\nu_{e^{-}}$ and $\bar{\nu}_{e}$-absorption on hot nuclei are given. The results of the numerical calculations are presented and discussed in Sec. III for the sample nuclei ${ }^{56} \mathrm{Fe}$ and ${ }^{82} \mathrm{Ge}$. For ${ }^{56} \mathrm{Fe}$ we compare the calculated ground-state and finitetemperature cross sections with available results from other approaches. Conclusions are drawn in Sec. IV. In an Appendix we prove in a model independent way that the principle of detailed balance is valid for chargechanging transitions if hot nuclei in the supernova environment are treated in the grand canonical ensemble.

\section{FORMALISM}

During the core-collapse phase of a supernovae explosion the temperature in the iron core is sufficiently high (a few $10^{9} \mathrm{~K}$ ) to establish an equilibrium of reactions mediated by the strong and electro-weak interaction [1]. Neglecting weak-interaction reactions, we can consider nuclei as open quantum systems in thermal equilibrium with heat and particle reservoirs and, hence, they can be described as a thermal grand canonical ensemble with temperature $T$ and proton and neutron chemical potentials $\lambda_{p}$ and $\lambda_{n}$, respectively. In TFD, such ensemble is represented by the thermal vacuum $|0(T)\rangle$, which is a temperature-dependent state in the extended Hilbert space [19]. The thermal vacuum is determined as the zero-energy eigenstate of the thermal Hamiltonian, $\mathcal{H}=H-\widetilde{H}$, and it satisfies the thermal state condition

$$
A|0(T)\rangle=\sigma_{A} \mathrm{e}^{\mathcal{H} / 2 T} \widetilde{A}^{\dagger}|0(T)\rangle .
$$

In the above equations $H$ is the original nuclear Hamiltonian (proton and neutron chemical potentials are included in $H$ ) and $\widetilde{H}$ is its tilde counterpart acting in the auxiliary Hilbert space; an operator $A$ acts in the physical Hilbert space, $\widetilde{A}$ is its tilde partner, and $\sigma_{A}$ is a phase factor. The thermal state condition guarantees that the expectation value $\langle 0(T)|A| 0(T)\rangle$ is equal to the grand canonical average of $A$. In this sense, relation (1) is equivalent to the Kubo-Martin-Schwinger condition for an equilibrium grand canonical density matrix [20].

External perturbations mediated by weak interaction induce transitions from the thermal vacuum to nonequilibrium states. Within the TDF such nonequilibrium states are given by the eigenstates of the thermal Hamiltonian $\mathcal{H}$. As follows from the definition of $\mathcal{H}$, each of its eigenstates with positive energy has a counterpart - the tilde-conjugate eigenstate - with negative but the same absolute value of energy. This gives the possibility to describe both endoergic and exoergic neutrino reactions with hot nuclei. In what follows we will refer to positive energy eigenstates as non-tilde states, and to negative energy eigenstates as tilde states.

\section{A. Thermal quasiparticle RPA}

In $[17,18]$ we have introduced the proton-neutron TQRPA method which allows for a treatment of charge-changing transitions in hot nuclei induced by (anti)neutrino absorption. For the sake of completeness, let us briefly recall the method. 
Within the TQRPA, nonequilibrium states of a hot nucleus caused by an external perturbation are treated as phonon-like excitations on the thermal vacuum

$$
\begin{aligned}
& \left|Q_{J M i}\right\rangle=Q_{J M i}^{\dagger}|0(T)\rangle, \\
& \left|\widetilde{Q}_{J M i}\right\rangle=\widetilde{Q}_{J M i}^{\dagger}|0(T)\rangle,
\end{aligned}
$$

where we denote $\widetilde{Q}_{\overline{J M} i}^{\dagger}=(-1)^{J-M} \widetilde{Q}_{J-M i}^{\dagger}$. Phonon excitations are considered as the normal modes of the thermal Hamiltonian:

$$
\mathcal{H} \simeq \sum_{J M i} \omega_{J i}(T)\left(Q_{J M i}^{\dagger} Q_{J M i}-\widetilde{Q}_{J M i}^{\dagger} \widetilde{Q}_{J M i}\right),
$$

while the thermal vacuum $|0(T)\rangle$ itself is the vacuum for the annihilation operators $Q_{J M i}, \widetilde{Q}_{J M i}$. Thus, within the TQRPA the problem of finding the excitation spectrum of a hot nucleus is reduced to the diagonalization of the thermal Hamiltonian in terms of phonon operators such that the respective phonon vacuum obeys the thermal state condition (1).

For charge-changing multipole transitions the phonon operators are defined as a linear superposition of creation and annihilation operators of proton-neutron thermal quasiparticle pairs [21]

$$
\begin{aligned}
Q_{J M i}^{\dagger}= & \sum_{j_{p} j_{n}}\left\{\psi_{j_{p} j_{n}}^{J i}\left[\beta_{j_{p}}^{\dagger} \beta_{j_{n}}^{\dagger}\right]_{M}^{J}+\widetilde{\psi}_{j_{p} j_{p}}^{J i}\left[\widetilde{\beta}_{\overline{J_{p}}}^{\dagger} \widetilde{\beta}_{\overline{J_{n}}}^{\dagger}\right]_{M}^{J}\right. \\
& +i \eta_{j_{p} j_{n}}^{J i}\left[\beta_{j_{p}}^{\dagger} \widetilde{\beta}_{\overline{J_{n}}}^{\dagger}\right]_{M}^{J}+i \widetilde{\eta}_{j_{p} j_{n}}^{J i}\left[\widetilde{\beta}_{\overline{J_{p}}}^{\dagger} \beta_{j_{n}}^{\dagger}\right]_{M}^{J} \\
& +\phi_{j_{p} j_{n}}^{J i}\left[\beta \overline{\bar{J}_{p}} \beta_{\overline{J_{n}}}\right]_{M}^{J}+\widetilde{\phi}_{j_{p} j_{n}}^{J i}\left[\widetilde{\beta}_{j_{p}} \widetilde{\beta}_{j_{n}}\right]_{M}^{J} \\
& \left.+i \xi_{j_{p} j_{n}}^{J i}\left[\beta_{\overline{J_{p}}} \widetilde{\beta}_{j_{n}}\right]_{M}^{J}+i \widetilde{\xi}_{j_{p} j_{n}}^{J i}\left[\widetilde{\beta}_{j_{p}} \beta_{\overline{J_{n}}}\right]_{M}^{J}\right\} .
\end{aligned}
$$

In turn, thermal quasiparticles are normal modes of the pairing part of the thermal Hamiltonian

$$
\mathcal{H}_{\text {pair }} \simeq \sum_{\tau} \sum_{j m}^{\tau} \varepsilon_{j}(T)\left(\beta_{j m}^{\dagger} \beta_{j m}-\widetilde{\beta}_{j m}^{\dagger} \widetilde{\beta}_{j m}\right),
$$

and their vacuum is the thermal vacuum in the BCS approximation. In the expression above, the notation $\sum^{\tau}$ implies a summation over neutron $(\tau=n)$ or proton $(\tau=p)$ single particle states only. Thermal quasiparticles are connected with Bogoliubov quasiparticles by so-called thermal transformation [22]

$$
\begin{aligned}
& \beta_{j m}^{\dagger}=x_{j} \alpha_{j m}^{\dagger}-i y_{j} \widetilde{\alpha}_{j m}, \\
& \widetilde{\beta}_{j m}^{\dagger}=x_{j} \widetilde{\alpha}_{j m}^{\dagger}+i y_{j} \alpha_{j m}, \quad\left(x_{j}^{2}+y_{j}^{2}=1\right) .
\end{aligned}
$$

The $(x, y)$-coefficients as well as the $(u, v)$-coefficients of the Bogoliubov transformations are found from the finitetemperature BCS equations (see [18] for more details). In accordance with the BCS theory [23, 24], the numerical solution of these equations yields vanishing pairing correlations above a certain critical temperature.

To clarify the physical meaning of different terms in (4), we note that the creation of a negative energy tilde thermal quasiparticle corresponds to the annihilation of a thermally excited Bogoliubov quasiparticle or, which is the same, to the creation of a quasihole state (see [18] for more details). Therefore, at finite temperature, single-particle charge-changing transitions involve excitations of three types: 1) two-quasiparticle excitations described by the operator $\beta_{j_{p}}^{\dagger} \beta_{j_{n}}^{\dagger}$ and having energy $\left.\varepsilon_{j_{p} j_{n}}^{(+)}=\varepsilon_{j_{p}}+\varepsilon_{j_{n}}, 2\right)$ one-quasiparticle one-quasihole excitations described by the operators $\beta_{j_{p}}^{\dagger} \widetilde{\beta}_{j_{n}}^{\dagger}, \widetilde{\beta}_{j_{p}}^{\dagger} \beta_{j_{n}}^{\dagger}$ and having energies $\varepsilon_{j_{p} j_{n}}^{(-)}=\varepsilon_{j_{p}}-\varepsilon_{j_{n}}$ and $-\varepsilon_{j_{p} j_{n}}^{(-)}$, respectively, and 3) two-quasihole excitations described by the operator $\widetilde{\beta}_{j_{p}}^{\dagger} \widetilde{\beta}_{j_{n}}^{\dagger}$ and having energy $-\varepsilon_{j_{p} j_{n}}^{(+)}$. The last two types are possible only at $T \neq 0$. Therefore, due to singleparticle transitions involving annihilation of thermally excited Bogoliubov quasiparticles, the phonon spectrum at finite temperature contains negative- and low-energy states which do not exist at zero temperature and these "new" phonon states can be interpreted as thermally unblocked transitions between nuclear excited states.

To find the energy and the wavefunctions of thermal phonons we apply the equation of motion method

$$
\left.\langle| \delta Q,\left[\mathcal{H}, Q^{\dagger}\right]\right]|\rangle=\omega(T)\left\langle\left|\left[\delta Q, Q^{\dagger}\right]\right|\right\rangle
$$

under two additional constraints: (a) the phonon vacuum obeys the thermal state condition (1), and (b) phonon operators obey Bose commutation relations. The first constraint yields the following relations between phonon amplitudes

$$
\begin{aligned}
& \left(\begin{array}{l}
\widetilde{\psi} \\
\widetilde{\phi}
\end{array}\right)_{j_{p} j_{n}}^{J i}=\frac{y_{j_{p}} y_{j_{n}}-\mathrm{e}^{-\omega_{J i} / 2 T} x_{j_{p}} x_{j_{n}}}{\mathrm{e}^{-\omega_{J i} / 2 T} y_{j_{p}} y_{j_{n}}-x_{j_{p}} x_{j_{n}}}\left(\begin{array}{c}
\phi \\
\psi
\end{array}\right)_{j_{p} j_{n}}^{J i}, \\
& \left(\begin{array}{l}
\widetilde{\eta} \\
\widetilde{\xi}
\end{array}\right)_{j_{p} j_{n}}^{J i}=\frac{y_{j_{p}} x_{j_{n}}-\mathrm{e}^{-\omega_{J i} / 2 T} x_{j_{p}} y_{j_{n}}}{\mathrm{e}^{-\omega_{J i} / 2 T} y_{j_{p}} x_{j_{n}}-x_{j_{p}} y_{j_{n}}}\left(\begin{array}{l}
\xi \\
\eta
\end{array}\right)_{j_{p} j_{n}}^{J i},
\end{aligned}
$$

while the last assumption is equivalent to averaging with respect to the BCS thermal vacuum in the equations of motion (7) and leads to an orthonormality condition for the amplitudes [18]. Thus, the phonon energies $\omega$ in Eq.(3) as well as the amplitudes $\psi, \widetilde{\psi}$, etc. in Eq.(4) are the solution of the proton-neutron TQRPA equations. Positive energy solutions correspond to non-tilde phonons in (3), while negative energy solutions correspond to tilde ones. Since both the energies of thermal quasiparticles and the interaction strengths between them are temperature dependent, the spectrum of thermal phonons turns out to be temperature dependent. However, it is significant that in the zero-temperature limit the described method reduces into the standard proton-neutron QRPA.

For a given multipole charge-changing transition operator $\mathcal{T}_{J}^{(\mp)}$ the transition probabilities (strengths) from the thermal vacuum to thermal one-phonon states are 
given by the following reduced matrix elements

$$
\begin{aligned}
& \Phi_{J i}^{(\mp)}=\left|\left\langle Q_{J i}\left\|\mathcal{T}_{J}^{(\mp)}\right\| 0(T)\right\rangle\right|^{2}, \\
& \widetilde{\Phi}_{J i}^{(\mp)}=\left|\left\langle\widetilde{Q}_{J i}\left\|\mathcal{T}_{J}^{(\mp)}\right\| 0(T)\right\rangle\right|^{2} .
\end{aligned}
$$

Here the symbol $(-)$ refers to neutron-to-proton transitions $\left(\beta^{-}\right.$channel), while $(+)$refers to proton-to-neutron transitions $\left(\beta^{+}\right.$channel). It can be shown that if the transition operators $\mathcal{T}_{J}^{(-)}$and $\mathcal{T}_{J}^{(+)}$differ only by the isospin operator, i.e., $\mathcal{T}_{J}^{(\mp)}=\mathcal{T}_{J} t_{\mp}$, the transition probabilities to tilde and non-tilde phonon states are connected as

$$
\widetilde{\Phi}_{J i}^{(\mp)}=\exp \left(-\frac{\omega_{J i}}{T}\right) \Phi_{J i}^{( \pm)} .
$$

In [15], a similar relation is obtained for charge-neutral transitions and it is referred to as the principle of detailed balance. However, in contrast to the case of chargeneutral transitions, the detailed balance relation (10) includes a phonon energy rather than a transition energy. The latter is the energy transferred to the parent nucleus and for charge-changing transitions is given by [25]

$$
\begin{aligned}
& E_{J i}^{(\mp)}=\omega_{J i} \mp \delta_{n p}, \\
& \widetilde{E}_{J i}^{( \pm)}=-E_{J i}^{(\mp)},
\end{aligned}
$$

where $\delta_{n p}=\Delta \lambda_{n p}+\Delta M_{n p}$, and $\Delta \lambda_{n p}$ is the difference between neutron and proton chemical potentials in the parent nucleus and $\Delta M_{n p}=1.293 \mathrm{MeV}$ is the neutronproton mass splitting. It is obvious that non-tilde (tilde) charge-changing phonon states do not necessarily correspond to upward (downward) transitions: due to $\delta_{n p}$, some non-tilde (tilde) phonon states may have negative (positive) transition energy. With Eq. (11), relation (10) is rewritten as

$$
\widetilde{\Phi}_{J i}^{(\mp)}=\exp \left(-\frac{E_{J i}^{( \pm)} \mp \delta_{n p}}{T}\right) \Phi_{J i}^{( \pm)} .
$$

Thus, for each $n \rightarrow p(p \rightarrow n)$ upward transition with energy $E$ there is an inverse downward transition $p \rightarrow n$ $(n \rightarrow p)$ with energy $-E$ and the respective transition probabilities are connected by (12). In the Appendix we show that in this form the detailed balance for chargechanging transitions in the ensemble of hot nuclei can be derived in a model independent way.

\section{B. Cross sections in supernova environments}

In the derivation of the (anti)neutrino absorption cross section for a hot nucleus under supernova conditions we follow the Walecka-Donnelly formalism [26, 27] which is based on the standard current-current form of the weak interaction Hamiltonian. After a multipole expansion of the weak nuclear current the temperature-dependent differential cross section for a transition from the thermal vacuum to a thermal one-phonon state is given by

$$
\begin{aligned}
\frac{d \sigma_{J i}\left(E_{\nu}, T\right)}{d \Omega} & =\frac{\left(G_{F} \cos \theta_{C}\right)^{2}}{\pi} p_{e}^{i} E_{e}^{i}\left\{\sigma_{C L}^{J}+\sigma_{T}^{J}\right\} \\
& \times F\left( \pm Z+1, E_{e}\right)\left(1-f\left(E_{e}\right)\right) .
\end{aligned}
$$

Here and below, the upper (lower) sign refers to the neutrino (antineutrino) cross section. In the above expression, $G_{F}$ is the Fermi constant for the weak interaction, $\theta_{C}$ is the Cabbibo angle, and $E_{e}$ and $p_{e}$ are the energy and momentum of the outgoing electron or positron.

In Eq. (13), the contributions, $\sigma_{C L}^{J}$, for the Coulomb and longitudinal components, and $\sigma_{T}^{J}$, for the transverse electric and magnetic components, are written as

$$
\begin{gathered}
\sigma_{C L}^{J}=(1+a \cos \Theta)\left|\left\langle J i\left\|\hat{M}_{J}\right\| 0(T)\right\rangle\right|^{2}+(1+\cos \Theta \\
\left.-2 b \sin ^{2} \Theta\right)\left|\left\langle J i\left\|\hat{L}_{J}\right\| 0(T)\right\rangle\right|^{2}+\left[\frac{E_{J i}}{q}(1+\cos \Theta)+c\right] \\
\times 2 \operatorname{Re}\left\langle J i\left\|\hat{L}_{J}\right\| 0(T)\right\rangle\left\langle J i\left\|\hat{M}_{J}\right\| 0(T)\right\rangle^{*}, \quad(1) \\
\sigma_{T}^{J}=\left(1-a \cos \Theta+b \sin ^{2} \Theta\right)\left[\left|\left\langle J i\left\|\hat{T}_{J}^{\mathrm{mag}}\right\| 0(T)\right\rangle\right|^{2}+\right. \\
\left.\left|\left\langle J i\left\|\hat{T}_{J}^{\mathrm{el}}\right\| 0(T)\right\rangle\right|^{2}\right] \mp\left[\frac{E_{\nu}+E_{e}^{i}}{q}(1-a \cos \Theta)-c\right] \\
\times 2 \operatorname{Re}\left\langle J i\left\|\hat{T}_{J}^{\mathrm{mag}}\right\| 0(T)\right\rangle\left\langle J i\left\|\hat{T}_{J}^{\mathrm{el}}\right\| 0(T)\right\rangle^{*}, \quad
\end{gathered}
$$

where $\Theta$ is the lepton scattering angle and the notation $|J i\rangle$ is used to denote both the non-tilde and the tilde thermal one-phonon states. In the former case the transition energy $E_{J i}=E_{J i}^{(\mp)}$, while in the latter case $E_{J i}=\widetilde{E}_{J i}^{(\mp)}$. Thus, the energy of the outgoing lepton is $E_{e}^{i}=E_{\nu}-E_{J i}$. The parameters $a, b$ and $c$ are obtained from the relations

$$
\begin{aligned}
& a=\frac{p_{e}}{E_{e}}=\sqrt{1-\left(\frac{m_{e} c^{2}}{E_{e}}\right)^{2}}, \\
& b=a^{2} \frac{E_{\nu} E_{e}}{q^{2}}, \quad c=\frac{\left(m_{e} c^{2}\right)^{2}}{q E_{e}},
\end{aligned}
$$

and the absolute value of the three-momentum transfer $q$ is given

$$
q=\sqrt{E_{J i}^{2}+2 E_{e} E_{\nu}(1-a \cos \Theta)-\left(m_{e} c^{2}\right)^{2}} .
$$

The multipole operators $\hat{M}_{J}, \hat{L}_{J}, \hat{J}_{J}^{\mathrm{el}}$, and $\hat{J}_{J}^{\mathrm{mag}}$ denote the charge, longitudinal, and transverse electric and magnetic parts of the hadronic current, respectively, as definedvin [26, 27]. For the vector, axial-vector, and pseudoscalar form-factors which describebthe internal structure of the nucleon we use parametrization from Ref. [28] (see also Ref. [29]).

For charged-current reactions, the cross section (13) must be corrected for the distortion of the outgoing lepton wave function by the Coulomb field of the residual (daughter) nucleus. The cross section can either be multiplied a Fermi function $F(Z, E)$ (see, e.g., Ref. [30]), 
or, at higher energies, the effect of the Coulomb field can be described by the effective momentum approximation (EMA) [31]. In the present study, by following the prescription from $[32,33]$, we choose an energy point in which both approaches predict the same values. Then the Fermi function is used below the point and the EMA is adopted above it.

Furthermore, in the supernova environment nuclei are surrounded by a nearly degenerate electron gas. Thus, neutrino absorption can be strongly reduced by Pauli blocking of the final electron states. The blocking factor $\left(1-f\left(E_{e}\right)\right)$ in Eq. (13) accounts for this effects, where $f\left(E_{e}\right)$ is the Fermi-Dirac distribution with temperature $T$ and the chemical potential $\mu_{e^{-}}$. The positron distribution is defined in the same way with $\mu_{e^{+}}=-\mu_{e^{-}}$. Therefore, we can neglect the blocking factor for antineutrino absorption.

The total cross section $\sigma\left(E_{\nu}, T\right)$, as a function of temperature and incoming (anti)neutrino energy, is obtained from the differential cross sections (13) by integrating over the scattering angle and summing over all possible final thermal one-phonon states

$$
\begin{aligned}
\sigma\left(E_{\nu}, T\right) & =2 \pi \sum_{J i} \int_{1}^{-1} \frac{d \sigma_{J i}}{d \Omega} d \cos \Theta \\
& =\sigma_{\mathrm{en}}\left(E_{\nu}, T\right)+\sigma_{\mathrm{ex}}\left(E_{\nu}, T\right) .
\end{aligned}
$$

Here, the total cross section is split into two parts: $\sigma_{\text {en }}\left(E_{\nu}, T\right)$ describes the endoergic neutrino absorption and includes only upward transitions $\left(E_{J i}>0\right)$, while $\sigma_{\text {ex }}\left(E_{\nu}, T\right)$ corresponds to the exoergic process associated with downward transitions $\left(E_{J i}<0\right)$. The contribution $\sigma_{\text {ex }}\left(E_{\nu}, T\right)$ dominates the cross section for vanishing neutrino energies, $E_{\nu} \approx 0$. It is apparent that for beta-stable nuclei the exoergic absorption is only possible at finite temperatures and due to transitions from thermally excited states.

For low-energy (anti)neutrinos, i.e., in the long wavelength limit $q \rightarrow 0$, the allowed $1^{+}$multipole operator reduces to the Gamow-Teller form

$$
\mathrm{GT}_{\mp}=g_{A} \vec{\sigma} t_{\mp},
$$

where $g_{A}=-1.2599$ [34] is the axial weak coupling constant. Considering only Gamow-Teller transitions and taking into account detailed balance according to (10), the cross section (18) can be written as

$$
\begin{aligned}
& \sigma\left(E_{\nu}, T\right)=\frac{\left(G_{F} \cos \theta_{C}\right)^{2}}{\pi} \\
& \quad \times\left\{\sum_{i}\left(E_{\nu}-E_{i}^{(\mp)}\right)^{2} \Phi_{i}^{(\mp)} F\left( \pm Z+1, E_{e}\right)\right. \\
& \left.\quad+\sum_{i}\left(E_{\nu}+E_{i}^{( \pm)}\right)^{2} \exp \left(-\frac{\omega_{i}}{T}\right) \Phi_{i}^{( \pm)} F\left( \pm Z+1, E_{e}\right)\right\}
\end{aligned}
$$

Here for simplicity the electron rest mass has been neglected, i.e. $E_{e} \approx p_{e}$, and the blocking factor for the outgoing lepton has been dropped. The matrix elements $\Phi_{i}^{(\mp)}$ denote the transition strength of the $\mathrm{GT}_{\mp}$ operator. The first term in Eq. (20) implies summation over non-tilde $1^{+}$states. Within the shell model approach this term reduces to the ground-state contribution (see Eq. (2) in Ref. [7]). However, in the TQRPA this term appears to be temperature dependent due to violation of Brink's hypothesis. The second term accounts for transitions to tilde states and it is an analog of the backresonance contribution within the shell-model approach. Here, we would like to stress that we cannot associate transitions to non-tilde (tilde) states with the endoergic (exoergic) absorption. As was mentioned above, due to $\delta_{n p}$ some non-tilde (tilde) states may correspond to downward (upward) transitions and, hence, contribute to $\sigma_{\mathrm{ex}}$ $\left(\sigma_{\mathrm{en}}\right)$.

\section{RESULTS AND DISCUSSION}

We employ the theoretical framework presented above to study thermal effects on the $\left(\nu_{e}, e^{-}\right)$and $\left(\bar{\nu}_{e}, e^{+}\right)$cross sections for two sample nuclei, ${ }^{56} \mathrm{Fe}$ and ${ }^{82} \mathrm{Ge}$. The iron isotope is among the most abundant nuclei at the early stage of the core-collapse, while the neutron-rich germanium isotope can be considered as the average nucleus at later stages [35].

To describe charge-changing transitions in a hot nucleus we use the same phenomenological nuclear Hamiltonian as in [18]. The Hamiltonian consists of spherically symmetric Woods-Saxon potentials for protons and neutrons, BCS pairing interactions, and separable multipole and spin-multipole residuals interaction in the particlehole channel. We neglect particle-particle interactions except for the BCS pairing forces. This Hamiltonian is usually referred to as the quasiparticle-phonon model (QPM) [36]. For the two nuclei considered, the parameters of the QPM Hamiltonian are fixed in the same manner as in $[15,18]$. Here we just mention that solving the BCS equations at zero temperature we get the following proton and neutron pairing gaps: $\Delta_{p(n)}=$ $1.57(1.36) \mathrm{MeV}$ for ${ }^{56} \mathrm{Fe}$ and $\Delta_{p(n)}=1.22(0.0) \mathrm{MeV}$ for ${ }^{82} \mathrm{Ge}$. Thus, the critical temperature $\left(T_{\mathrm{cr}} \approx 0.5 \Delta_{\tau}\right)$ when the pairing phase transition occurs is $T_{\mathrm{cr}} \approx 0.8 \mathrm{MeV}$ for the iron isotope and $T_{\mathrm{cr}} \approx 0.6 \mathrm{MeV}$ for the germanium isotope.

A separable form of the residual interaction allows us to reduce the TQRPA equations to a secular equation. The explicit form for charge-changing excitations as well as expressions for transition strengths (9) are given in [17, 18]. Moreover, it has been proved in Ref. [17] that the TQRPA fulfills the Ikeda sum rule for allowed Fermi and GT transitions. 


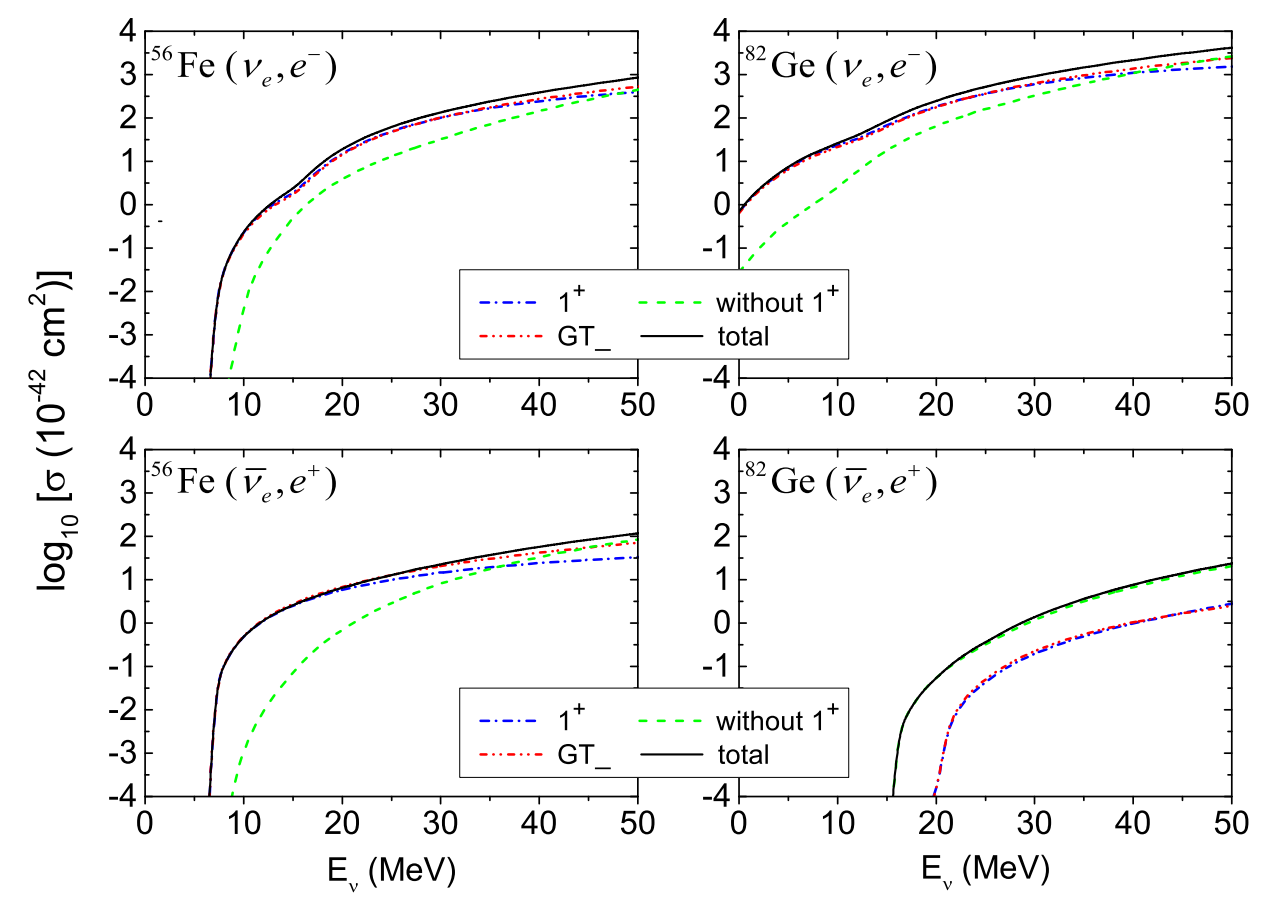

FIG. 1. (Color online) Cross sections for neutrino (upper panels) and antineutrino (lower panels) absorption reactions for ${ }^{56}$ Fe and ${ }^{82} \mathrm{Ge}$. The total cross sections (solid lines) include contributions of the $J^{\pi}=0^{ \pm}-3^{ \pm}$multipoles. The dashed lines show the cross sections without the $1^{+}$contribution. The dash-dotted lines correspond to the $1^{+}$contributions calculated with the full momentum-dependent transition operator whereas the $1^{+}$contributions calculated with the Gamow-Teller operator (19) are shown by the dash-double dotted lines.

\section{A. Ground-state cross sections}

As in [15], before proceeding to discuss thermal effects on neutrino-nucleus absorption cross sections, we consider the ground-state $(T=0)$ cross sections for ${ }^{56} \mathrm{Fe}\left(\nu_{e}, e^{-}\right),{ }^{56} \mathrm{Fe}\left(\bar{\nu}_{e}, e^{+}\right),{ }^{82} \mathrm{Ge}\left(\nu_{e}, e^{-}\right)$, and ${ }^{82} \mathrm{Ge}\left(\bar{\nu}_{e}, e^{+}\right)$ reactions and compare the our results with those available from other theoretical studies. In Fig. 1, the calculated total cross sections are shown as functions of the incident (anti)neutrino energy $E_{\nu}$. We find that for $E_{\nu} \leq 100 \mathrm{MeV}$ the reactions considered are dominated by the multipole transitions $J^{\pi} \leq 3^{\mp}$, while contributions from higher multipoles are only a few percent of the total cross sections.

For ${ }^{56} \mathrm{Fe}$ the cross sections increase sharply as $E_{\nu}$ approaches the reaction threshold $Q_{-}=4.56 \mathrm{MeV}$ for $\nu_{e}$-absorption and $Q_{+}=4.71 \mathrm{MeV}$ for $\bar{\nu}_{e}$-absorption $\left(Q_{\mp}=M_{f}+m_{e} c^{2}-M_{i}\right.$, where $M_{i, f}$ are the masses of the parent and daughter nuclei). As the neutron number increases, the threshold energy for $\nu_{e}$-absorption decreases. For the neutron-rich nucleus ${ }^{82} \mathrm{Ge}$ the $Q_{-}$-value becomes negative $\left(Q_{-}=-4.71 \mathrm{MeV}\right)$ allowing neutrino absorption for all energies. Contrary to this, for the $\left(\bar{\nu}_{e}, e^{+}\right)$ reactions the $Q_{+}$-values become less favorable with increasing neutron excess. The antineutrino has to overcome a noticeable threshold $\left(Q_{+}=13.58 \mathrm{MeV}\right)$ to be absorbed by ${ }^{82} \mathrm{Ge}$ and the corresponding cross section is considerably lower in comparison to the neutrino one.
In addition to the total cross sections, contributions from the $1^{+}$multipole channel are shown in Fig. 1 . Referring to the plots in Fig. 1 for the ${ }^{56} \mathrm{Fe}\left(\nu_{e}, e^{-}\right)$, ${ }^{56} \mathrm{Fe}\left(\bar{\nu}_{e}, e^{+}\right),{ }^{82} \mathrm{Ge}\left(\nu_{e}, e^{-}\right)$reactions the cross sections are dominated by allowed $1^{+}$transitions for energies up to $E_{\nu}=30 \mathrm{MeV}$, with contributions from other multipoles being much smaller. In the ${ }^{82} \mathrm{Ge}\left(\bar{\nu}_{e}, e^{+}\right)$cross section, however, the $1^{+}$contribution is negligible and forbidden transitions dominate the reaction. We find that $1^{-}$and $2^{-}$transitions mainly contribute to the cross section for $E_{\nu}<50 \mathrm{MeV}$.

In Fig. 1 we also analyze the effect of the full $q$ dependent $1^{+}$transition operator instead of its longwavelength limit. As was mentioned above, in the latter case the $1^{+}$transition operator reduces to the GamowTeller operator (19) and the ground-state cross section is given by the first term in Eq. (20). It should be noted that to make a comparison with the shell-model calculations $[37,38]$ more transparent, we use the same quenching factor for the axial weak coupling constant, $g_{A}^{*}=0.74 g_{A}$. By comparing in Fig. 1 the $1^{+}$and GamowTeller contributions to the cross sections, we conclude that for energies $E_{\nu} \leq 30 \mathrm{MeV}$ the application of the GT operators instead of the $q$-dependent $1^{+}$operator is fully justified. Therefore, we conclude that the lowenergy ground-state cross sections for the ${ }^{56} \mathrm{Fe}\left(\nu_{e}, e^{-}\right)$, ${ }^{56} \mathrm{Fe}\left(\bar{\nu}_{e}, e^{+}\right),{ }^{82} \mathrm{Ge}\left(\nu_{e}, e^{-}\right)$reactions are completely dominated by $\mathrm{GT}$ transitions. In contrast, all $\mathrm{GT}_{+}$transi- 
TABLE I. Total ${ }^{56} \mathrm{Fe}\left(\nu_{e}, e^{-}\right)$cross sections for selected neutrino energies $E_{\nu}$. The present QRPA results (second column) are compared with those from [39] and with the hybrid approach results [37]. The cross sections are given in units of $10^{-42} \mathrm{~cm}^{2}$, with exponents given in parentheses.

\begin{tabular}{cccc}
\hline \hline$E_{\nu}(\mathrm{MeV})$ & QRPA & SkQRPA[39] & Hybrid[37] \\
\hline \hline 10 & $2.39(-1)$ & $3.63(+0)$ & $6.61(-1)$ \\
15 & $2.35(+0)$ & $1.73(+1)$ & $6.45(+0)$ \\
20 & $1.91(+1)$ & $5.26(+1)$ & $2.93(+1)$ \\
25 & $6.19(+1)$ & $1.25(+2)$ & $7.33(+1)$ \\
30 & $1.34(+2)$ & $2.33(+2)$ & $1.40(+2)$ \\
40 & $3.88(+2)$ & $5.44(+2)$ & $3.71(+2)$ \\
50 & $8.47(+2)$ & $9.83(+2)$ & $7.98(+2)$ \\
60 & $1.58(+3)$ & $1.67(+3)$ & $1.38(+3)$ \\
70 & $2.61(+3)$ & $2.59(+3)$ & $2.42(+3)$ \\
80 & $3.95(+3)$ & $3.73(+3)$ & $3.60(+3)$ \\
90 & $5.53(+3)$ & $5.07(+3)$ & $4.98(+3)$ \\
100 & $7.26(+3)$ & $6.60(+3)$ & $6.52(+3)$ \\
\hline \hline
\end{tabular}

TABLE II. Same as Table I, but for the ${ }^{56} \mathrm{Fe}\left(\bar{\nu}_{e}, e^{+}\right)$reaction.

\begin{tabular}{cccc}
\hline \hline$E_{\nu}(\mathrm{MeV})$ & QRPA & SkQRPA[39] & Hybrid[38] \\
\hline \hline 10.0 & $4.92(-1)$ & $2.95(+0)$ & $0(+0)$ \\
12.5 & $1.34(+0)$ & $6.09(+0)$ & $0(+0)$ \\
15.0 & $2.59(+0)$ & $1.03(+1)$ & $2(+0)$ \\
20.0 & $6.48(+0)$ & $2.17(+1)$ & $7(+0)$ \\
25.0 & $1.28(+1)$ & $3.74(+1)$ & $1.6(+1)$ \\
30.0 & $2.26(+1)$ & $5.74(+1)$ & $3.0(+1)$ \\
40.0 & $5.71(+1)$ & $1.20(+2)$ & $8.4(+1)$ \\
50.0 & $1.17(+2)$ & $2.09(+2)$ & $1.81(+2)$ \\
\hline \hline
\end{tabular}

tions of are essentially blocked in ${ }^{82} \mathrm{Ge}$. For nuclei with $Z<40$ and $N>40$ blocking occurs because the valence protons are in the $p f$ shell, while the valence neutrons occupy already the next major shell ( $s d g$ shell). In the next section it will be shown, however, that thermal effects unblock $\mathrm{GT}_{+}$transitions in ${ }^{82} \mathrm{Ge}$ and for typical supernova temperatures the low-energy ${ }^{82} \mathrm{Ge}\left(\bar{\nu}_{e}, e^{+}\right)$cross sections are also dominated by the GT contributions.

In Tables I and II, we compare the calculated groundstate cross sections for (anti)neutrino absorption by ${ }^{56} \mathrm{Fe}$ to those obtained from self-consistent QRPA calculations with Skyrme forces [39] and with the hybrid approach (large-scale shell-model calculations for GT contributions and RPA for other multipoles) [37, 38]. As is seen, the three models, although based on different microscopic pictures, predict rather similar neutrino absorption cross sections. We note, however, that for low energies $\left(E_{\nu} \leq 40 \mathrm{MeV}\right)$ the present results are closer to the hybrid approach results than those of Ref. [39]. It is evident that the reason why the QRPA and the hybrid approach cross sections are systematically lower than those of the Skyrme based calculations at $E_{\nu} \leq 40 \mathrm{MeV}$

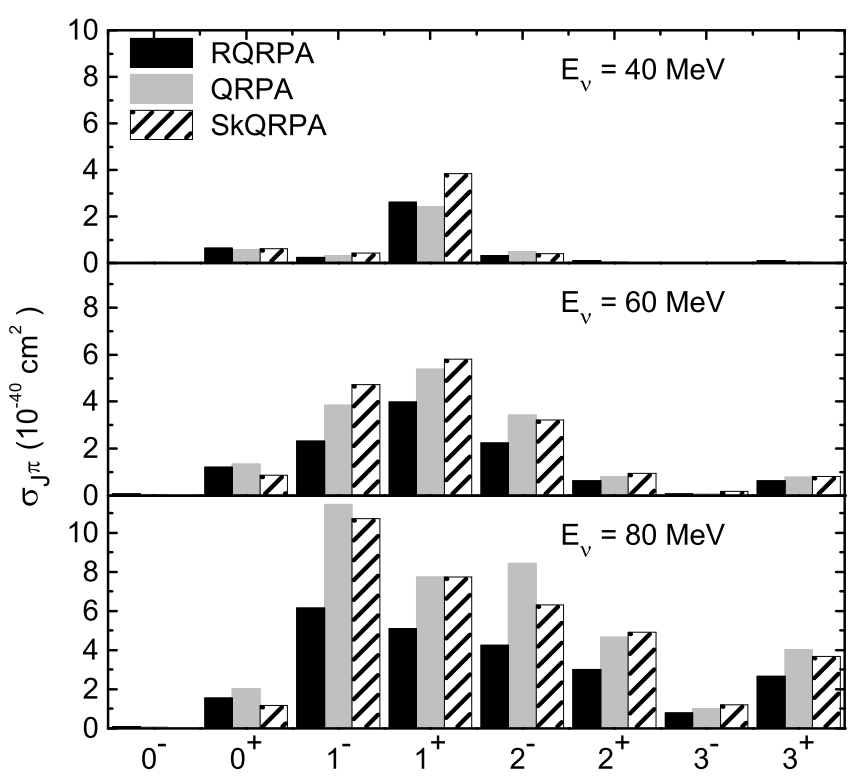

FIG. 2. Contribution of the multipole transitions $J^{\pi}=$ $0^{ \pm}-3^{ \pm}$to the cross section for the ${ }^{56} \mathrm{Fe}\left(\nu_{e}, e^{-}\right){ }^{56}$ Co reaction at $E_{\nu}=40,60,80 \mathrm{MeV}$. The present QRPA results are compared with those obtained from RQRPA [41] and SkQRPA [39] calculations.

is caused by differences in the GT - strength distributions. Although the authors of Ref. [39] do not provide the $\mathrm{GT}_{-}$distribution in ${ }^{56} \mathrm{Fe}$, it seems that the Skyrme based calculations result in a larger strength below the $\mathrm{GT}_{\text {- }}$ resonance compared to the other two approaches. Other possible reasons for the discrepancy could be a lower energy of the GT _ resonance and a larger total GT strength obtained from the calculations with the Skyrme interaction.

Experimental results for neutrino absorption cross sections are rather limited. The KARMEN Collaboration has measured the flux averaged ${ }^{56} \mathrm{Fe}\left(\nu_{e}, e^{-}\right)^{56} \mathrm{Co}$ cross section for the neutrino spectrum from the muon decay at rest and obtains $\langle\sigma\rangle=[251 \pm 83$ (stat.) \pm 42 (syst.) $] \times$ $10^{-42} \mathrm{~cm}^{2}[40]$. Our result, $\langle\sigma\rangle=223 \times 10^{-42} \mathrm{~cm}^{2}$, is in excellent agreement with the experimental value. Note, that the hybrid approach predicts $\langle\sigma\rangle=240 \times$ $10^{-42} \mathrm{~cm}^{2}$ [37], while the QRPA calculations with Skyrme forces gives $\langle\sigma\rangle=352 \times 10^{-42} \mathrm{~cm}^{2}$ [39].

For ${ }^{56} \mathrm{Fe}$ a detailed demonstration of the most important multipole contributions to neutrino absorption cross section is presented in Fig. 2 at three neutrino energies, $E_{\nu}=40,60$, and $80 \mathrm{MeV}$. As expected, at relatively low neutrino energies $\left(E_{\nu} \lesssim 40 \mathrm{MeV}\right)$ the dominant contribution to the cross section originates from $1^{+}$transitions. With increasing $E_{\nu}$, however, contributions from other multipole transitions become important. In particular, at $E_{\nu}=80 \mathrm{MeV}$, the dominant contribution comes from $1^{-}$transitions, but other multipoles, e.g., $1^{+}, 2^{-}, 2^{+}$, and $3^{+}$, also play an important role.

In Fig. 2, we also compare the calculated multipole 
decomposition of the cross section for ${ }^{56} \mathrm{Fe}$ with those from the SkQRPA calculations [39] and the relativistic QRPA (RQRPA) calculations [41]. As is evident from the figure, the latter model predicts somewhat smaller cross section at high neutrino energies $\left(E_{\nu} \gtrsim 60 \mathrm{MeV}\right)$, whereas we observe an excellent agreement between the results of the present QRPA and the SkQRPA. Specifically, in accordance with Ref. [39, 41], we find that $0^{+}$allowed transitions only marginally contribute to the ${ }^{56} \mathrm{Fe}\left(\nu_{e}, e^{-}\right)$ reaction. This finding is true for other three reactions and for finite temperatures as well. For this reason, in the discussion below, we will always imply $1^{+}$multipole channels when referring the allowed transitions.

\section{B. Finite temperatures}

In discussing $\nu_{e}$ and $\bar{\nu}_{e}$ absorption reactions under supernova conditions we will follow the line of our recent work [15] and consider first thermal effects on the strength distribution of GT transitions which dominate the reactions at low energies.

In Fig. 3, we display on a logarithmic scale the GT and $\mathrm{GT}_{+}$distributions in ${ }^{56} \mathrm{Fe}$ calculated for the groundstate $(T=0)$ and at three stellar temperatures above the critical one, $T=0.86 \mathrm{MeV}\left(10^{10} \mathrm{~K}\right), 1.29 \mathrm{MeV}(1.5 \times$ $\left.10^{10} \mathrm{~K}\right)$, and $1.72 \mathrm{MeV}\left(2 \times 10^{10} \mathrm{~K}\right)$. These temperatures roughly correspond to three stages in the collapse evolution [9]. We emphasize that the distributions are plotted with the bare GT operators $\vec{\sigma} t_{\mp}$ as functions of the transition energy (11). For each temperature we show the value of $\delta_{n p}$ in Eq. (11) as well as the total transition strengths $S_{-}$and $S_{+}$. Note that $S_{-}$and $S_{+}$values calculated for the ground-state satisfy the Ikeda sum rule $S_{-}-S_{+}=3(N-Z)$ (a small deviation is caused by the incompleteness of our single-particle basis) but noticeably overestimate the experimental data $\left(S_{-}=9.9 \pm 2.4\right.$ [42], $\left.S_{+}=2.9 \pm 0.3[43]\right)$. This overestimation is common for any QRPA calculations of GT strength and is remedied by a quenched value for the axial weak coupling constant $g_{A}$. One might notice that our QRPA calculations fairly well reproduce the experimental centroid energies for both $\mathrm{GT}_{+}[43]$ and $\mathrm{GT}_{-}[42]$ distributions in ${ }^{56} \mathrm{Fe}$. In this respect the present calculations are consistent with the large-scale shell-model calculations [44]. Of course, in contrast to the LSSM approach, the QRPA cannot recover all nuclear correlations needed to reproduce the fragmentation of the GT strength.

For ${ }^{56} \mathrm{Fe}$, due to a relatively small absolute value of $\delta_{n p}$, the major part of the upward (downward) GT strength corresponds to transitions to non-tilde (tilde) phonon states. According to our QRPA calculations, the principal contribution to the $\mathrm{GT}_{-}\left(\mathrm{GT}_{+}\right)$ strength in ${ }^{56} \mathrm{Fe}$ comes from the $1 f_{7 / 2} \rightarrow 1 f_{5 / 2}$ neutronto-proton (proton-to-neutron) single-particle transition which forms a resonance peak at $E \approx 14 \mathrm{MeV}(\approx 6 \mathrm{MeV})$. Because the Brink hypothesis is not valid within the TQRPA, we observe an evolution of the GT resonances

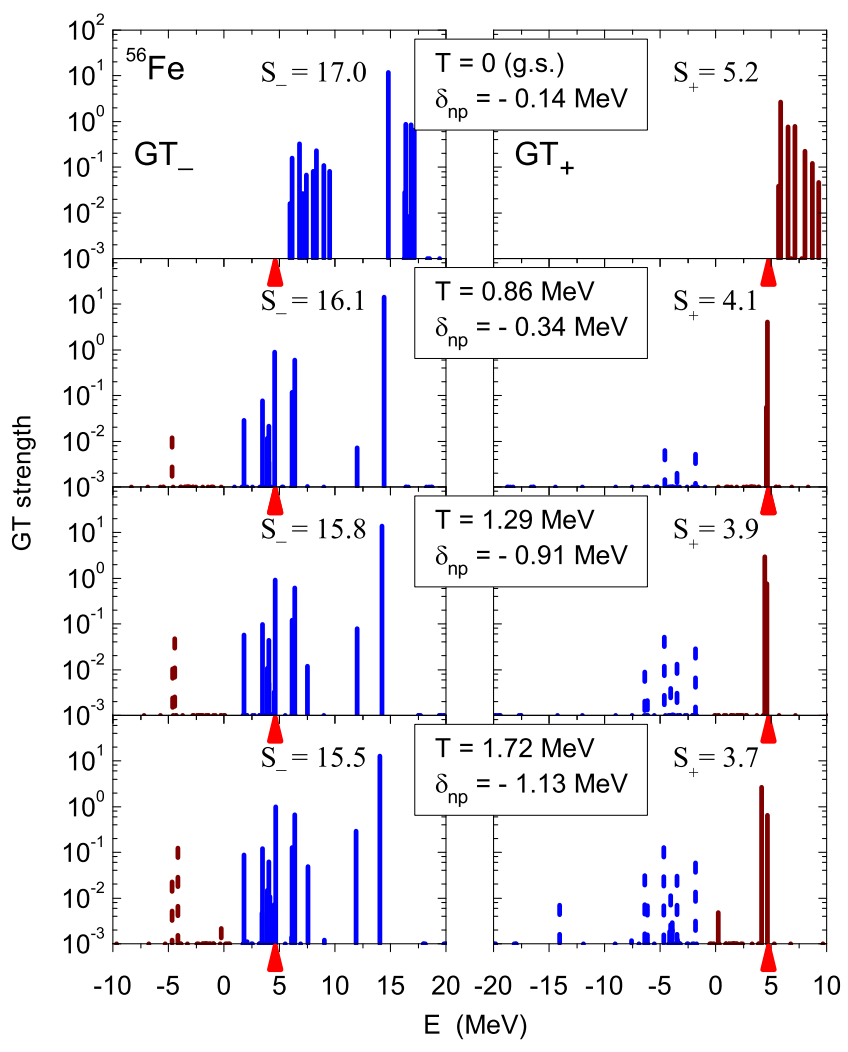

FIG. 3. (Color online) Temperature evolution of GT- (left panels) and $\mathrm{GT}_{+}$(right panels) strength distributions for ${ }^{56} \mathrm{Fe}$ vs transition energy. The solid (dashed) lines refer to transitions to non-tilde (tilde) thermal one-phonon states. The arrows indicate the ground-state reaction thresholds for neutrino $\left(Q_{-}=4.56 \mathrm{MeV}\right)$ and antineutrino $\left(Q_{+}=4.71 \mathrm{MeV}\right)$ absorption.

with temperature. Namely, when the temperature is increased to $0.86 \mathrm{MeV}$, the transition energy is lowered by $\sim 1.2 \mathrm{MeV}$ for the $\mathrm{GT}_{-}$resonance and $\sim 2.1 \mathrm{MeV}$ for the $\mathrm{GT}_{+}$resonance. This decrease in energy is mainly attributed to the vanishing of pairing correlations: at temperatures above the critical one no extra energy is needed to break a proton (neutron) Cooper pair and as a consequence the $\mathrm{GT}_{+}\left(\mathrm{GT}_{-}\right)$resonance moves to lower energies. However, not only pairing effects lead to downward shift of the GT resonances. It can be easily seen from the structure of the TQRPA equations [17] that quasiparticle thermal occupations factors will appear which screen the interaction term. Due to the thermal blocking of the proton-neutron repulsive residual interaction, a further increase in temperature to $1.72 \mathrm{MeV}$ decreases the $\mathrm{GT}_{-}$and $\mathrm{GT}_{+}$resonances in ${ }^{56} \mathrm{Fe}$ by $\sim 0.3 \mathrm{MeV}$ and $\sim 0.5 \mathrm{MeV}$, respectively. As was mentioned in the introduction, the observed downward shift of the GT strength is not present in large-scale shell-model calculations which are partially based on Brink's hypothesis. In contrast, the finite-temperature relativistic QRPA calculations in Ref. [45] and shell-model Monte-Carlo calculations in Ref. [10] show similar features for the changes of 
the GT resonance energy.

Finite temperature also affects the low-energy GT strength in ${ }^{56} \mathrm{Fe}$ : due to the gradual disappearance of pairing it partially shifts below the ground-state reaction threshold and becomes more fragmented. The fragmentation arises from the thermal smearing of the nuclear Fermi surface that unblocks low-energy particleparticle and hole-hole $\mathrm{GT}_{-}$transitions. Here, particle (hole) denotes a state above (below) the Fermi level. Moreover, in accordance with detailed balance (12), the temperature rise exponentially increases the strength of negative-energy downward transitions. Referring to the figure, the dominant part of the downward GT - strength around $E \approx-4.1 \mathrm{MeV}$ originates from a transition inverse to the $\mathrm{GT}_{+}$resonance, that is due to the transition from the thermally populated neutron orbit $1 f_{5 / 2}$ to the lower lying proton orbit $1 f_{7 / 2}$. In contrast, transitions inverse to the $\mathrm{GT}_{-}$resonance are suppressed by the energy-dependent exponent in Eq. (12) and the GT + downward strength is dominated by transitions inverse to low-energy GT _ transitions. It should be mentioned that although thermal effects unblock some new GT transitions, the total $\mathrm{GT}_{\mp}$ strength in ${ }^{56} \mathrm{Fe}$ slightly decreases with temperature. Nevertheless, the TQRPA preserves the Ikeda sum rule.

It is obvious that the violation of Brink's hypothesis should affect the downward GT strength. In [15], this influence was studied thoroughly for charge-neutral GT transitions in ${ }^{56} \mathrm{Fe}$ by comparing the GT running sums derived with and without Brink's hypothesis. Applying the same method, we find that both the shift of the GT + resonance to lower energies and the thermal unblocking of low-lying $\mathrm{GT}_{-}$strength enhance the $\mathrm{GT}_{-}$and $\mathrm{GT}_{+}$ downward strengths in ${ }^{56} \mathrm{Fe}$.

Fig. 4 shows the temperature evolution of the GT strength function for the neutron-rich nucleus ${ }^{82} \mathrm{Ge}$. The distributions are displayed at the same temperatures as those for ${ }^{56} \mathrm{Fe}$ in Fig. 3. As evident from the figure, some GT strength associated with non-tilde (tilde) phonon states appears at negative (positive) transition energies which is due to a large value of $\delta_{n p}$. The temperature rise affects the $\mathrm{GT}_{-}$and $\mathrm{GT}_{+}$distributions in a different way. For the $\mathrm{GT}_{-}$distribution, a temperature increase essentially affects only the downward part, yielding some new strength below $-10 \mathrm{MeV}$. By comparing the left and right panels in Fig. 4, we conclude that this thermally unblocked strength corresponds to transitions inverse to the $\mathrm{GT}_{+}$resonance. The $\mathrm{GT}_{-}$resonance is weakly sensitive to thermal effects. By increasing the temperature to $T=1.72 \mathrm{MeV}$, the excitation energy gets only lowered by $\sim 0.5 \mathrm{MeV}$. Since there are no neutron pairing correlations in ${ }^{82} \mathrm{Ge}$, this lowering is solely caused by the softening of the residual interaction.

In contrast, thermal effect are significant for the $\mathrm{GT}_{+}$ resonance and they clearly demonstrate a violation of the Brink hypothesis within the TQRPA. Namely, with rising temperature the resonance peak shifts by $\sim 7 \mathrm{MeV}$ to lower energies and the total $\mathrm{GT}_{+}$strength shows a non-

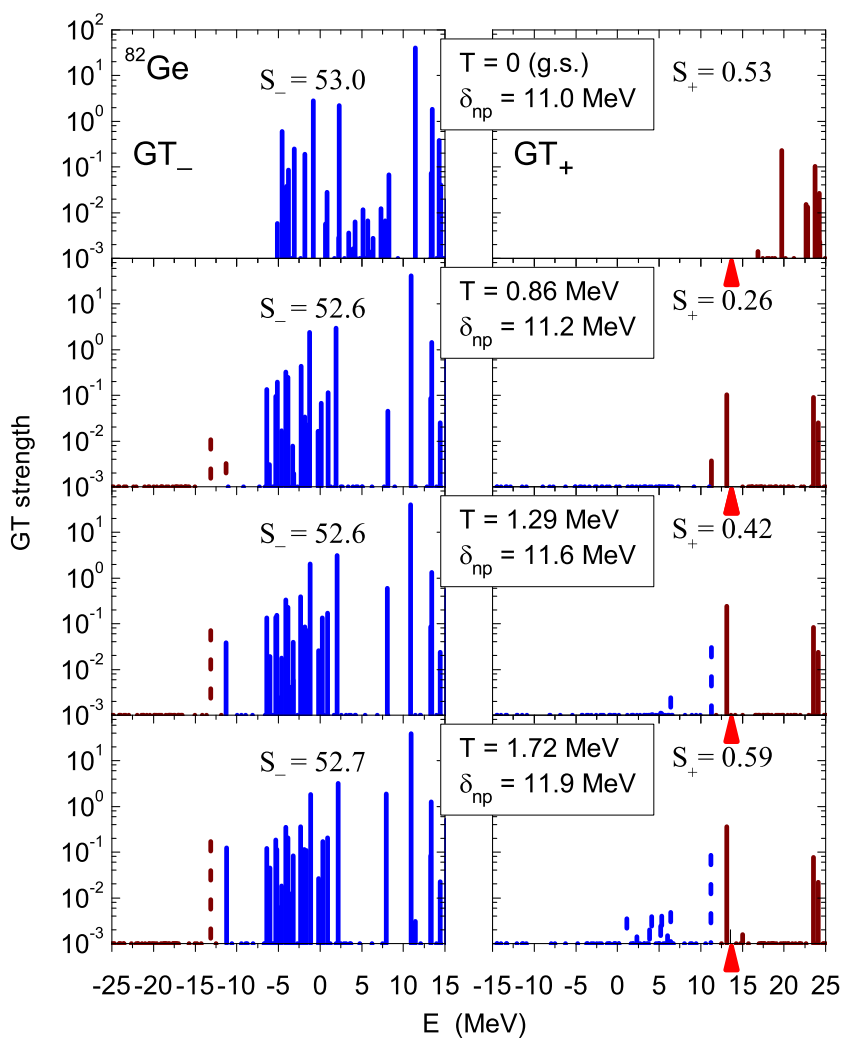

FIG. 4. (Color online) Same as Fig. 3 but for ${ }^{82}$ Ge. Due to Pauli blocking of allowed $p \rightarrow n$ transitions, the total GT strength is many times larger than the $\mathrm{GT}_{+}$strength and $S_{-} \approx 3(N-Z)=54$.

monotomic dependence: after an initial decrease, it gradually increases. In [18], a detailed investigation within the TQRPA approach was performed of thermal effects on the $\mathrm{GT}_{+}$strength in the neutron-rich nucleus ${ }^{76} \mathrm{Ge}$. The conclusions remain valid for ${ }^{82} \mathrm{Ge}$, as well. Briefly, for neutron-rich nuclei with $N>40$ and $Z<40$ the Independent Particle Model predicts that all GT $\mathrm{GT}_{+}$transitions of valence protons are Pauli blocked due to the complete occupation of the $p f$ neutron orbits. However, both thermal excitations and pairing correlations promote protons to the $s d g$ shell and make possible $1 g_{9 / 2}^{p} \rightarrow 1 g_{7 / 2}^{n}$ particleparticle transition in ${ }^{82} \mathrm{Ge}$ possible. The important point is that the transition energy drastically decreases with temperature. Namely, at $T<T_{\text {cr }}$, when pairing correlations are important, the transition energy is given by $\varepsilon_{1 g_{7 / 2}^{n}}+\varepsilon_{1 g_{9 / 2}^{p}}+\delta_{p n} \approx 20 \mathrm{MeV}$, while at $T>T_{\text {cr }}$, when thermal effects become significant, it is given by $\varepsilon_{1 g_{7 / 2}^{n}}-\varepsilon_{1 g_{9 / 2}^{p}}+\delta_{p n} \approx 13 \mathrm{MeV}$. In addition, the transition strength below the GT + resonance becomes increasingly unblocked with temperature. In particular, a strong peak due to the $1 f_{7 / 2}^{p} \rightarrow 1 f_{5 / 2}^{n}$ hole-hole transition appears at $E \approx 11 \mathrm{MeV}$. Both unblocking mechanisms are severely suppressed in the vicinity of the critical temperature $\left(T_{\mathrm{cr}} \approx 0.6 \mathrm{MeV}\right.$ in $\left.{ }^{82} \mathrm{Ge}\right)$, i.e. when pairing correlations vanish while thermal effects are not yet suf- 

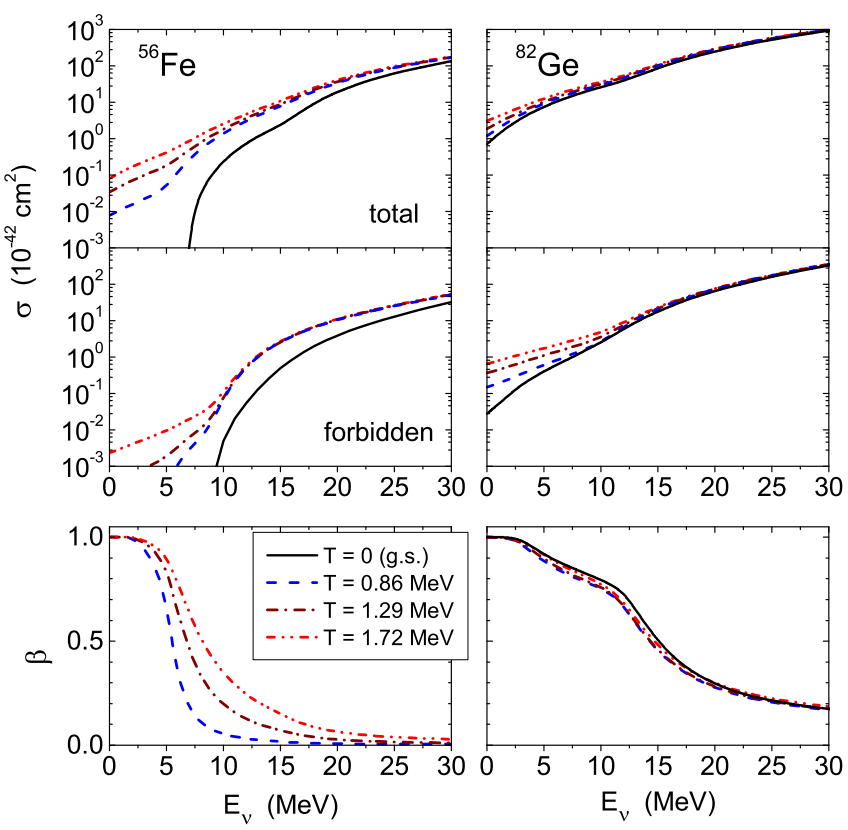

FIG. 5. (Color online) (Upper panels) Total neutrino absorption cross sections for ${ }^{56} \mathrm{Fe}$ and ${ }^{82} \mathrm{Ge}$ at three different temperatures relevant for core collapse. For comparison, the ground-state cross sections are also shown. (Middle panels) Contributions from forbidden transitions to the cross sections. (Lower panels) Temperature dependence of the ratio of exoergic absorption to the reaction cross section.

ficiently strong to significantly occupy the $1 g_{9 / 2}$ proton orbit or unblock the $1 f_{5 / 2}$ neutron orbit. Therefore, the total $\mathrm{GT}_{+}$strength decreases at $T \approx T_{\mathrm{cr}}$. The thermal effects on the $\mathrm{GT}_{+}$strength in neutron-rich nuclei discussed here were predicted in [35] and also confirmed in [45] by calculations based on the finite-temperature relativistic QRPA. We also note that, due to the large $\delta_{n p}$, downward $\mathrm{GT}_{+}$transitions appears to be highly suppressed in ${ }^{82} \mathrm{Ge}$.

Let us now illustrate the influence of the thermal effects discussed above on the neutrino and antineutrino absorption by ${ }^{56} \mathrm{Fe}$ and ${ }^{82} \mathrm{Ge}$. In Fig. 5, we compare the $\left(\nu_{e}, e^{-}\right)$ground-state cross sections with those calculated at the three core-collapse temperatures. For energies $E_{\nu}<30 \mathrm{MeV}$, which are typical for supernova neutrinos, the cross sections are dominated by allowed

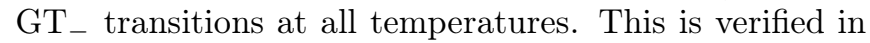
the middle panels of Fig. 5, where the overall contribution of forbidden transitions to the finite temperature cross sections is given. Although the forbidden cross section increases with temperature, it contributes less than $30 \%$ all the way up to $E_{\nu}=30 \mathrm{MeV}$. The lower panels of Fig. 5 show the ratio of exoergic absorption to the reaction cross section

$$
\beta\left(E_{\nu}, T\right)=\frac{\sigma_{\mathrm{ex}}\left(E_{\nu}, T\right)}{\sigma\left(E_{\nu}, T\right)},
$$

where $\sigma_{\mathrm{ex}}\left(E_{\nu}, T\right)$ only accounts for negative-energy downward transitions induced by neutrino absorption.

When considering the ${ }^{56} \mathrm{Fe}\left(\nu_{e}, e^{-}\right)$cross section, we observe that thermal effects are unimportant for $E_{\nu}>$ $20 \mathrm{MeV}$, i.e. when incoming neutrinos have sufficiently large energy to excite the $\mathrm{GT}_{-}$resonance. Note, that a downward shift of the $\mathrm{GT}_{\text {- }}$ resonance only marginally affects the cross section at such high neutrino energies. Thermal effects become pronounced though for lower neutrino energies since finite temperature removes the reaction threshold and significantly enhances the cross section. For energies $E_{\nu}<5 \mathrm{MeV}$, the ratio $\beta>0.5$ and, hence, the observed enhancement is mostly caused by downward $\mathrm{GT}_{-}$transitions from nuclear excited states. Moreover, for $E_{\nu} \approx 0$ the thermally unblocked downward transitions completely dominate the reaction, increasing the cross section by more than an order of magnitude when the temperature rises from $0.86 \mathrm{MeV}$ to $1.72 \mathrm{MeV}$. The role of the exoergic absorption decreases with increasing neutrino energy and for intermediate energies, $5 \mathrm{MeV}<E_{\nu}<20 \mathrm{MeV}$, thermal effects on the $\mathrm{GT}_{-}$resonance and its low-energy tail become important for the cross section enhancement.

Referring to the right panels in Fig. 5 it is shown that the ${ }^{82} \mathrm{Ge}\left(\nu_{e}, e^{-}\right)$cross section to a much lesser extent depends on temperature than that for the ${ }^{56} \mathrm{Fe}\left(\nu_{e}, e^{-}\right)$ reaction. This result can be understood as follows. In ${ }^{82} \mathrm{Ge}$, due to the negative $Q_{-}$-value, the downward $\mathrm{GT}_{-}$ transitions dominate the ground-state reaction up to $E_{\nu} \approx 15 \mathrm{MeV}$. As discussed above, the GT _ distribution in ${ }^{82} \mathrm{Ge}$ is little affected by the temperature rise, which merely causes some additional strength at $E<-10 \mathrm{MeV}$. This thermally unblocked downward strength becomes competitive with the ground-state contribution only at rather low energies $\left(E_{\nu} \lesssim 5 \mathrm{MeV}\right)$. As a result, thermal effects on the ${ }^{82} \mathrm{Ge}\left(\nu_{e}, e^{-}\right)$reaction are noticeably milder than in the previous case and a temperature rise from $T=0$ to $1.72 \mathrm{MeV}$ enhances the low-energy cross section only by a factor of about four. This observation is in line with Ref. [7], where it was shown that the thermal enhancement of the neutrino absorption cross section is reduced the more neutron-rich the nucleus is.

The results for $\bar{\nu}_{e}$-absorption are shown in Fig. 6. For the ${ }^{56} \mathrm{Fe}\left(\bar{\nu}_{e}, e^{+}\right)$reaction the cross section demonstrates the same trend as discussed above for the neutrino absorption by ${ }^{56} \mathrm{Fe}$ : (i) The gap in the cross section disappears and the low-energy cross section increases with temperature. (ii) with increasing $E_{\nu}$ the cross sections at different temperatures converge, i.e., thermal effects become unimportant. From the lower-left panel we conclude that a significant enhancement of the low-energy cross section relative to ground-state calculations comes from the increasing contribution of downward GT - transitions from nuclear excited states. The ratio $\beta$ gradually reduces at $E_{\nu}>5 \mathrm{MeV}$, since at those energies the excitation of the $\mathrm{GT}_{+}$resonance becomes possible. However, at $T=1.72 \mathrm{MeV}$ the exoergic component of the cross section appears to be comparable with the endoergic one up to $E_{\nu} \approx 15 \mathrm{MeV}$. 

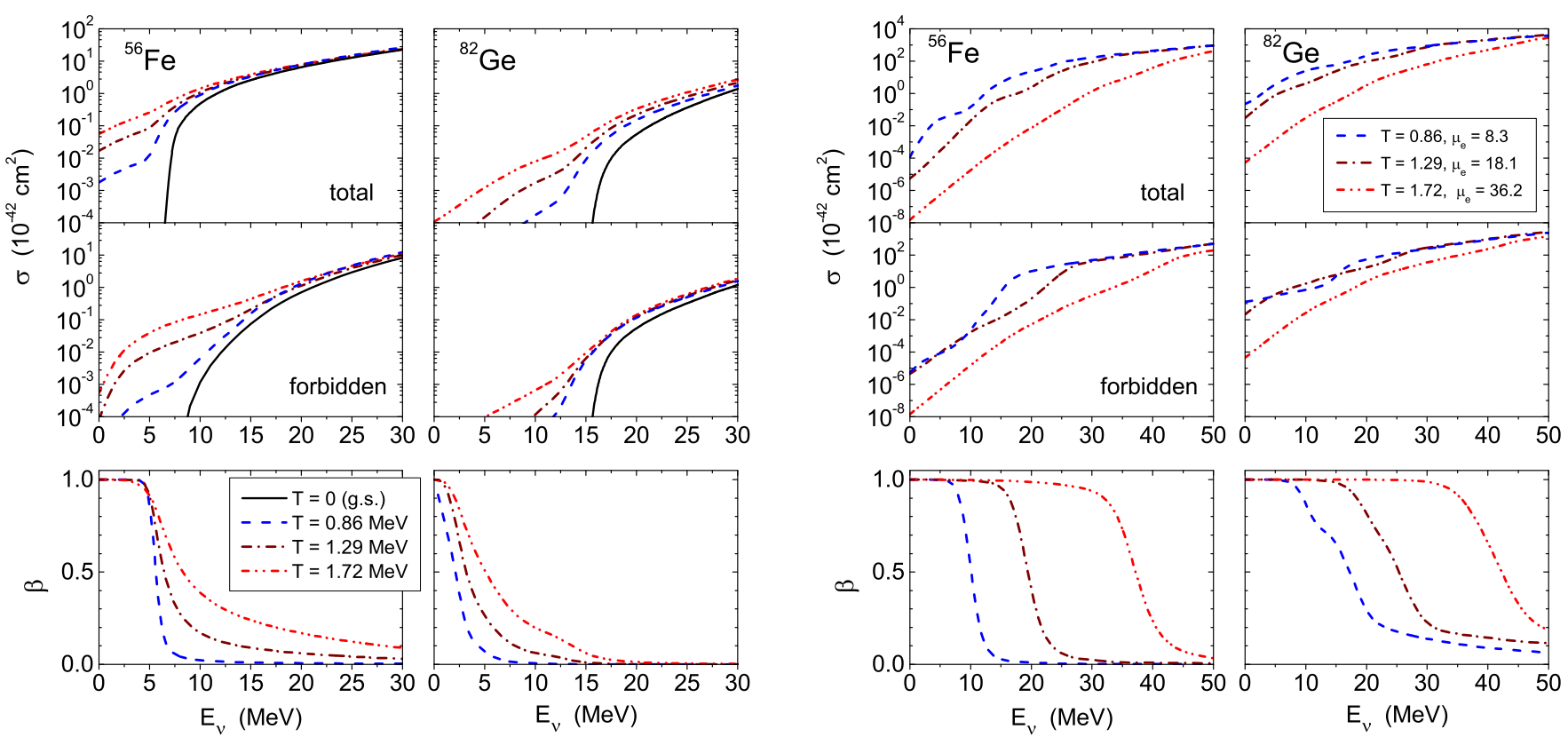

FIG. 6. (Color online) The same as in Fig. 5, but for the antineutrino absorption reaction.
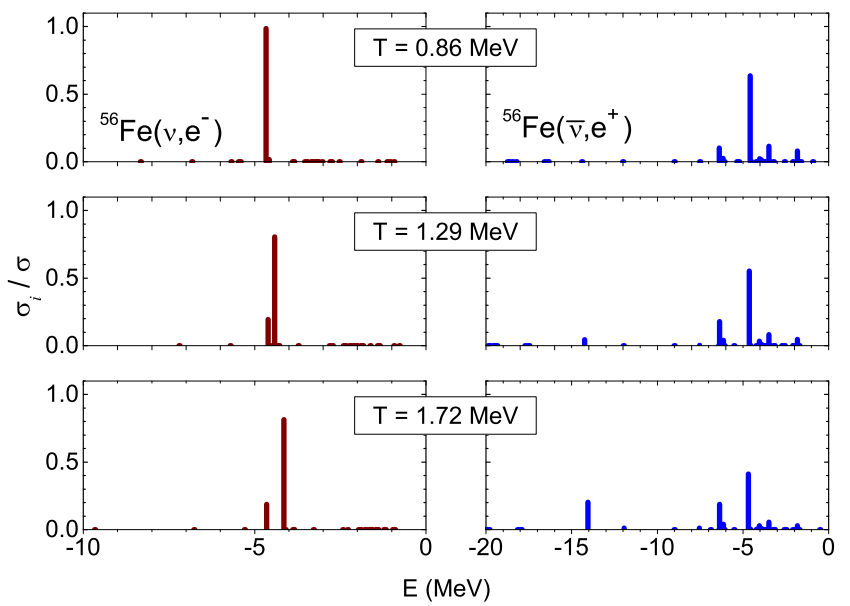

FIG. 7. (Color online) Relative contribution $\sigma_{i} / \sigma$ of the $i$ th negative-energy GT state to the finite-temperature cross section at $E_{\nu}=0$.

It should be noted that although the calculated ${ }^{56} \mathrm{Fe}\left(\nu_{e}, e^{-}\right)$and ${ }^{56} \mathrm{Fe}\left(\bar{\nu}_{e}, e^{+}\right)$cross sections show the same trend, the observed thermal enhancement at low energies is caused by different types of downward transitions. To show that, we have calculated the relative contribution $\sigma_{i} / \sigma$ of different negative-energy GT states to the cross section at $E_{\nu}=0$. The results are depicted in Fig. 7. As indicated in the figure, the $\nu$-absorption is completely dominated by the thermally unblocked transition inverse to the $\mathrm{GT}_{+}$resonance. For the $\bar{\nu}$-absorption, however, the main contribution to the reaction is given by transitions inverse to the $\mathrm{GT}_{-}$low-energy strength, while the "inverse" GT - resonance is suppressed by the

FIG. 8. (Color online) The same as in Fig. 5, but now including the outgoing electron blocking

Boltzmann factor in Eq. (20). Only at $T=1.72 \mathrm{MeV}$, the "inverse" GT _ resonance gives a noticeable contribution to the absorption of low-energy antineutrinos.

As illustrated in the right panels of Fig. 6, the lowenergy ${ }^{82} \mathrm{Ge}\left(\bar{\nu}_{e}, e^{+}\right)$cross section at finite temperature is also predominantly mediated by $\mathrm{GT}_{+}$transitions. $\mathrm{Al}-$ though the cross section remains substantially smaller as compared with the other three reactions considered, it demonstrates a strong temperature dependence for antineutrinos with $E_{\nu}<15 \mathrm{MeV}$, i.e. below the groundstate reaction threshold. Since the downward GT + transitions are suppressed in ${ }^{82} \mathrm{Ge}$, the cross section enhancement reflects the thermal unblocking of the upward $\mathrm{GT}_{+}$strength. Because of the thermal unblocking, the energy below which the $\mathrm{GT}_{+}$contribution is more than half of the total cross section shifts to higher values: at $T=0.86 \mathrm{MeV}$ this energy about $15 \mathrm{MeV}$, at $T=1.29 \mathrm{MeV}$ it is about $20 \mathrm{MeV}$, and at $T=1.72 \mathrm{MeV}$ it is about $25 \mathrm{MeV}$. For higher energies forbidden transitions dominate the process like at $T=0$ and the crosssection depends only weakly on temperature.

As was mentioned above, during the core contraction neutrino absorption by nuclei is hindered by Pauli blocking of the phase space for the outgoing electron. To study this effect within the TQRPA, following Ref. [7], we have calculated the $\nu$-absorption cross sections by introducing a blocking factor $\left(1-f\left(E_{e}\right)\right)$ defined at three different sets of temperature and chemical potential $\left(\mu_{e}\right.$ in $\mathrm{MeV}):\left(T, \mu_{e}\right)=(0.86,8.3),(1.29,18.1)$, and $(1.72,36.2)$. The results are shown in Fig. 8 for the ${ }^{56} \mathrm{Fe}\left(\nu_{e}, e^{-}\right)$and ${ }^{82} \mathrm{Ge}\left(\nu_{e}, e^{-}\right)$reactions. As can be seen, the neutrino absorption cross sections are drastically reduced due to electron blocking in the final state. Moreover, as the chem- 


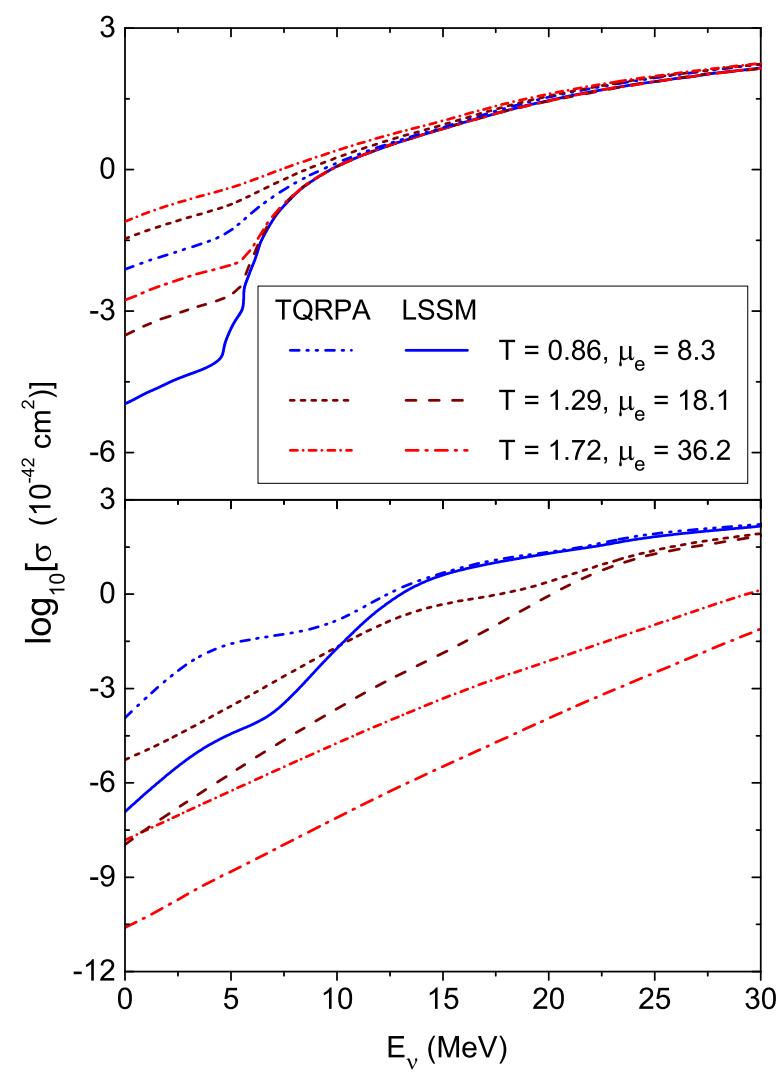

FIG. 9. (Color online) Comparison of the cross sections of neutrino absorption on the hot nucleus ${ }^{56} \mathrm{Fe}$ calculated within the TQRPA and the LSSM approach (see Ref. [7], Fig. 1). The cross sections in the upper (lower) panel are derived without (with) the final-state electron blocking. The respective temperatures and chemical potentials are given in $\mathrm{MeV}$.

ical potential increases faster than the temperature the cross sections decrease with temperature. It is obvious that the absorbtion due to de-excitation of thermally excited states is less affected by the Pauli blocking since the outgoing electron gains energy. This is clearly demonstrated by the plots in the lower panels of Fig. 8 where the relative contribution of negative-energy transitions is shown. We thus conclude that owing to blocking effect negative energy transitions dominate the cross sections up to neutrino energies $E_{\nu} \gtrsim \mu_{e}$. Another consequence of the blocking is that downward $1 \hbar \omega$ forbidden transitions become important with increasing $\mu_{e}$. Without Pauli blocking their contribution in negligible due to a small Boltzmann weight. However, as shown in the middle panels of Fig. 8, for $\left(T, \mu_{e}\right)=(1.29,18.1)$ and $(1.72,36.2)$ their contribution is comparable or even larger than those of the allowed transitions. According to our calculations this downward forbidden contribution is mainly due to $0^{-}, 1^{-}, 2^{-}$transitions.

In Fig. 9, the ${ }^{56} \mathrm{Fe}\left(\nu_{e}, e^{-}\right)$cross sections are displayed together with the results of the large-scale shell-model calculations (see Fig. 1 in Ref. [7]). Even if the general behaviour of the cross sections as functions of the neu- trino energy and temperature is in agreement in both approaches, the TQRPA results are noticeable larger than the LSSM ones but the discrepancy reduces with increasing neutrino energies. To understand the cause of the discrepancy, let us consider first the cross sections calculated neglecting the final-state electron blocking. For $E_{\nu}<5 \mathrm{MeV}$ the TQRPA cross sections exceed the LSSM values by two to three orders of magnitude. For such low energies, in both approaches, the neutrino absorption on ${ }^{56} \mathrm{Fe}$ is dominated by $\mathrm{GT}_{-}$downward transitions from thermally excited states. The differences in the description of such transitions explain the differences between the TQRPA and shell-model results. As the TQRPA is based on the grand canonical ensemble, the upward and downward $\mathrm{GT}_{ \pm}$strength are connected by the detailed balance. As shown in Fig. 7, the absorption of low-energy neutrinos on ${ }^{56} \mathrm{Fe}$ is dominated by the downward $\mathrm{GT}_{-}$transition inverse to the $\mathrm{GT}_{+}$resonance. This corresponds to an excitation energy $\omega_{i} \approx 6 \mathrm{MeV}$ in the Boltzmann factor of Eq. (20). Within the shellmodel calculations, downward transitions are included by back-resonances, that is by inverting the $\mathrm{GT}_{+}$strength distribution of the daughter nucleus. In [7], the backresonances are built on the lowest states of ${ }^{56} \mathrm{Co}$ and the bulk of the back-resonance strength in ${ }^{56} \mathrm{Fe}$ is located at an excitation energy of $E_{i} \approx 7-9 \mathrm{MeV}$. This excitation energy is somewhat higher than $\omega_{i}$. Moreover, within the TQRPA the downward strength concentrates in a single state, while the shell model GT + strength for ${ }^{56} \mathrm{Co}$ is highly fragmented owing to multinucleon correlations [46]. It is clear that both these factors suppress the contribution of downward transitions within the LSSM. To see whether the TQRPA reliably predicts the strength of negative-energy transitions, one must go beyond the TQRPA. For a separable residual interaction used here this can be done following the method developed within the QPM, that is by taking phonon coupling into account. On the other hand we should note that due to violation of Brink's hypothesis some backresonances built on high-lying excited states of ${ }^{56}$ Co may be located at the same energies as those built on the nuclear ground and low-lying states. Due to an increasing density of states, the contribution of such back-resonance states may be substantial and, therefore, their inclusion into the shell-model calculations may improve the agreement between the TQRPA and shell-model results. For $5<E_{\nu}<15 \mathrm{MeV}$, thermally unblocked low-energy GT transitions come into play, and since such transitions do not appear within the shell-model based calculation they also cause the excess of the TQRPA cross sections over the LSSM ones. At $E_{\nu}>15 \mathrm{MeV}, \nu$-absorption is dominated by the strong transition involving the $\mathrm{GT}_{-}$resonance. With increasing $E_{\nu}$, the cross section becomes insensitive to the energy dependence of the GT distribution and depends only on the total GT - strength. As a result we observe excellent agreement between the results of both approaches.

Comparing the cross sections calculated with Pauli 
blocking for the outgoing electron (see the lower panel of Fig. 9), we note that in [7], only allowed GT - transitions are taken into account when calculating neutrino absorption cross section. As is shown in Fig. 8, if the Pauli blocking is taken into account, forbidden downward transition become important with increasing temperature and electron chemical potential. It is apparent that such transitions along with the above discussed reasons lead to larger values for the TQRPA cross sections as compared to the shell-model results.

\section{CONCLUSION}

In this work, we have studied thermal effects on the (anti)neutrino absorption for hot nuclei in supernova environments. For this purpose, we have employed the proton-neutron QRPA extended to finite temperatures within the TFD formalism. As an example, cross sections were calculated for ${ }^{56} \mathrm{Fe}$ and ${ }^{82} \mathrm{Ge}$ in the temperature range from $T=0$ to $1.72 \mathrm{MeV}$ by taking into account the relevant charge-exchange transitions $J^{\pi}=0^{ \pm}, 1^{ \pm}, 2^{ \pm}$, and $3^{ \pm}$.

A detailed analysis of thermal effects was performed for allowed GT transitions which dominate the cross sections for $E_{\nu}<30 \mathrm{MeV}$ neutrinos. Since the TQRPA does not support the Brink hypothesis, new peaks appear in the $\mathrm{GT}_{\mp}$ strength function at finite temperature due to transitions from the excited states. Moreover, thermal effects shift the GT resonance centroids to lower energies and this effect appears more strongly for the $\mathrm{GT}_{+}$strength in ${ }^{82} \mathrm{Ge}$. The downward transitions from nuclear excited states were included in our calculations through detailed balance. The validity of detailed balance for charge-exchange transitions is a consequence of the grand canonical treatment of hot nuclei.

We have found that thermal effects on the GT strength enhance the absorption cross sections for low-energy (anti)neutrinos by several orders of magnitude. This enhancement is mainly due to increasing contributions of downward transitions from excited states. However, in the supernova environment the electron chemical potential increases more rapidly than temperature. As a result, if the electron blocking in the final state is taken into account, the neutrino cross sections are drastically reduced.

Although our calculations reveal the same thermal effects as the shell-model calculation, the calculated lowenergy cross sections for ${ }^{56} \mathrm{Fe}$ exceed the shell-model values by two to three orders of magnitude. One of the possible reason for this discrepancy is that the TQRPA underestimates multinucleon correlations which are responsible for the GT strength fragmentation. On the other hand, the inclusion of back-resonances built on highly excited daughter states into shell-model calculations may also improve the agreement between the TQRPA and shellmodel results.

Since the TQRPA is not restricted by iron-group nu- clei, it has some advantages over shell-model calculations. To enhance its reliability and predictive power several improvements could be made. First of all, to account for multiconfigurational effects, the coupling of thermal charge-exchange phonons with more complex (e.g., twophonon) configurations should be included into the approach. At zero temperature this problem was considered within the QPM $[47,48]$ by exploiting a separable form of the residual interaction. The other direction of the improvement is to combine the TQRPA method with self-consistent QRPA calculations based on either the relativistic or Skyrme nuclear energy density functionals. Recently, such calculations were performed at zero temperature [39, 41]. With a separable approximation for the Skyrme interaction [49] it will be possible calculate the phonon coupling at $T \neq 0$ within a self-consistent theory. This is planned for the future.

\section{ACKNOWLEDGMENTS}

We are greatly indebted to Prof. G. Martínez-Pinedo for helpful discussions and important comments on this paper. This work was supported by the HeisenbergLandau Program.

\section{Appendix}

Here we show that the detailed balance condition in the form (12) can be derived in a model independent way. When considering a grand canonical ensemble of hot nuclei, the probability to find the $i$ th excited state of a nucleus with $Z$ protons and $N$ neutrons is given by

$$
P\left(\varepsilon_{i}, A_{N}^{Z}\right)=\left(2 J_{i}+1\right) \exp \left\{-\frac{\varepsilon_{i}-\lambda_{n} N-\lambda_{p} Z}{T}\right\} \mathcal{Z}^{-1}
$$

where $\mathcal{Z}$ is the partition function of the grand canonical ensemble and $J_{i}$ is the angular momentum. Notice that the excitation energies $\varepsilon_{i}$ are counted from the energy of noninteracting nucleons, i.e., $\varepsilon_{0}$ is a groundstate binding energy and the chemical potentials $\lambda_{n, p}$ do not include nucleon rest mass. Let us now introduce the temperature-dependent strength function for chargeexchange transitions as a thermal average of all transition strengths (probabilities) from states in the parent nucleus to states in the daughter nucleus:

$$
\Phi^{(\mp)}(E)=\sum_{Z, N} \sum_{i, f} P\left(\varepsilon_{i}, A_{N}^{Z}\right) S_{i f}^{(\mp)} \delta\left(E-Q_{i f}^{(\mp)}\right) .
$$

Here $Q_{i f}^{(\mp)}=\varepsilon_{f}-\varepsilon_{i} \mp \Delta M_{n p}$ and

$$
S_{i f}^{(\mp)}=\left|\left\langle f, A_{N \mp 1}^{Z \pm 1}\left\|\mathcal{T}^{(\mp)}\right\| i, A_{N}^{Z}\right\rangle\right|^{2}
$$

is the reduced transition strength between states $i$ and $f$ in the parent and daughter nuclei, respectively. In the 
above equations the upper sign corresponds to $n \rightarrow p$ transitions, while the lower sign refers to $p \rightarrow n$ transitions. The transition energy $E$ can be both positive and negative.

For the transition operators $\mathcal{T}^{(-)}$and $\mathcal{T}^{(+)}$, which differ only by the isospin operator, the respective transition strengths, $S_{i f}^{(-)}$and $S_{f i}^{(+)}$, are connected by detailed balance through

$$
\left(2 J_{i}+1\right) S_{i f}^{(-)}=\left(2 J_{f}+1\right) S_{f i}^{(+)} .
$$

Combining this result with Eq. (A.2), we get the following relationship between the strength functions for $n \rightarrow p$ and $p \rightarrow n$ transitions in the thermal grand canonical ensemble

$$
\Phi^{( \pm)}(-E)=\Phi^{(\mp)}(E) \exp \left\{-\frac{E \mp\left(\Delta \lambda_{n p}+\Delta M_{n p}\right)}{T}\right\} .
$$

This relation is exactly the same as derived within the TQRPA approach for charge-changing transitions in hot nuclei.
[1] H.-T. Janka, K. Langanke, A. Marek, G. MartínezPinedo, and B. Müller, Physics Reports 442, 38 (2007), the Hans Bethe Centennial Volume 1906-2006.

[2] M. Liebendoerfer, (2004), astro-ph/0405029.

[3] A. Mezzacappa, (2004), astro-ph/0410085.

[4] W. C. Haxton, Phys. Rev. Lett. 60, 1999 (1988).

[5] S. W. Bruenn and W. C. Haxton, Astrophys. J. 376, 678 (1991).

[6] G. M. Fuller and B. S. Meyer, Astrophys. J. 376, 701 (1991).

[7] J. Sampaio, K. Langanke, and G. Martínez-Pinedo, Physics Letters B 511, 11 (2001).

[8] J. Sampaio, K. Langanke, G. Martínez-Pinedo, and D. Dean, Physics Letters B 529, 19 (2002).

[9] A. Juodagalvis, K. Langanke, G. Martínez-Pinedo, W. Hix, D. Dean, and J. Sampaio, Nuclear Physics A 747, 87 (2005).

[10] P. B. Radha, D. J. Dean, S. E. Koonin, K. Langanke, and P. Vogel, Phys. Rev. C 56, 3079 (1997).

[11] A. A. Dzhioev and A. I. Vdovin, International Journal of Modern Physics E 18, 1535 (2009).

[12] Y. TakahashI and H. Umezawa, International Journal of Modern Physics B 10, 1755 (1996).

[13] H. Umezawa, H. Matsumoto, and M. Tachiki, Thermo field dynamics and condensed states (North-Holland Pub. Co., 1982).

[14] I. Ojima, Annals of Physics 137, 1 (1981).

[15] A. A. Dzhioev, A. I. Vdovin, J. Wambach, and V. Y. Ponomarev, Phys. Rev. C 89, 035805 (2014).

[16] A. Dzhioev, A. Vdovin, V. Ponomarev, and J. Wambach, Physics of Atomic Nuclei 74, 1162 (2011).

[17] A. Dzhioev, A. Vdovin, V. Ponomarev, and J. Wambach, Physics of Atomic Nuclei 72, 1320 (2009).

[18] A. A. Dzhioev, A. I. Vdovin, V. Y. Ponomarev, J. Wambach, K. Langanke, and G. Martínez-Pinedo, Phys. Rev. C 81, 015804 (2010).

[19] The correspondence between the thermo-field dynamics and the so-called superoperator formalism is discussed in [50]. The latter is used by one of the authors (A.D.) to study nonequilibrium transport phenomena (see, e.g., [51]).

[20] R. Kubo, Journal of the Physical Society of Japan 12, 570 (1957); P. C. Martin and J. Schwinger, Phys. Rev. 115, 1342 (1959).

[21] In Eq. (4), [ $]_{M}^{J}$ denotes the coupling of two single-particle angular momenta $j_{p}, j_{n}$ to the total angular momen- tum $J$.

[22] Note that we use Ojima's[14] complex form of the thermal transformation.

[23] A. L. Goodman, Nuclear Physics A 352, 30 (1981).

[24] O. Civitarese, G. Dussel, and R. P. J. Perazzo, Nuclear Physics A 404, 15 (1983).

[25] At $T=0$, the transition energy corresponds to the final state energy measured from parent-nucleus ground state.

[26] J. D. Walecka, in Muon Physics V2: Weak Interactions, edited by V. W. Hughes and C. S. Wu (Elsevier Science, 1975) p. 113.

[27] T. W. Donnelly and R. D. Peccei, Physics Reports 50, 1 (1979).

[28] S. Singh, Nuclear Physics B - Proceedings Supplements 112, 77 (2002).

[29] H. Đapo and N. Paar, Phys. Rev. C 86, 035804 (2012).

[30] K. Langanke and G. Martínez-Pinedo, Nuclear Physics A 673, 481 (2000).

[31] J. Engel, Phys. Rev. C 57, 2004 (1998).

[32] C. Volpe, N. Auerbach, G. Colò, T. Suzuki, and N. Van Giai, Phys. Rev. C 62, 015501 (2000).

[33] N. Paar, D. Vretenar, T. Marketin, and P. Ring, Phys. Rev. C 77, 024608 (2008).

[34] I. S. Towner, in Symmetries and Fundamental Interactions in Nuclei, edited by W. C. Haxton and E. M. Henley (World Scientific Publishing Company, Incorporated, 1995) p. 183.

[35] J. Cooperstein and J. Wambach, Nuclear Physics A 420, 591 (1984).

[36] V. G. Soloviev, Theory of atomic nuclei: quasiparticles and phonons (Taylor \& Francis, 1992).

[37] E. Kolbe and K. Langanke, Phys. Rev. C 63, 025802 (2001).

[38] J. Toivanen, E. Kolbe, K. Langanke, G. Martínez-Pinedo, and P. Vogel, Nuclear Physics A 694, 395 (2001).

[39] R. Lazauskas and C. Volpe, Nuclear Physics A 792, 219 (2007).

[40] R. Maschuw, Progress in Particle and Nuclear Physics 40, 183 (1998).

[41] N. Paar, T. Suzuki, M. Honma, T. Marketin, and D. Vretenar, Phys. Rev. C 84, 047305 (2011).

[42] J. Rapaport, T. Taddeucci, T. Welch, C. Gaarde, J. Larsen, D. Horen, E. Sugarbaker, P. Koncz, C. Foster, C. Goodman, C. Goulding, and T. Masterson, Nuclear Physics A 410, 371 (1983).

[43] S. El-Kateb, K. P. Jackson, W. P. Alford, R. Abegg, R. E. 
Azuma, B. A. Brown, A. Celler, D. Frekers, O. Häusser, R. Helmer, R. S. Henderson, K. H. Hicks, R. Jeppesen, J. D. King, G. G. Shute, B. M. Spicer, A. Trudel, K. Raywood, M. Vetterli, and S. Yen, Phys. Rev. C 49, 3128 (1994).

[44] E. Caurier, K. Langanke, G. Martínez-Pinedo, and F. Nowacki, Nuclear Physics A 653, 439 (1999).

[45] Y. F. Niu, N. Paar, D. Vretenar, and J. Meng, Phys. Rev. C 83, 045807 (2011).

[46] K. Langanke and G. Martínez-Pinedo, Physics Letters B 453, 187 (1999).
[47] V. A. Kuzmin and V. G. Soloviev, Journal of Physics G: Nuclear Physics 10, 1507 (1984).

[48] V. A. Kuzmin and V. G. Soloviev, Journal of Physics G: Nuclear Physics 11, 603 (1985).

[49] N. Van Giai, C. Stoyanov, and V. V. Voronov, Phys. Rev. C 57, 1204 (1998).

[50] M. Schmutz, Zeitschrift für Physik B Condensed Matter 30, 97 (1978).

[51] A. A. Dzhioev and D. S. Kosov, Journal of Physics: Condensed Matter 24, 225304 (2012). 